OPEN ACCESS

Citation: Mayur Dhondiram Nandikar (2021) The genus Salacia (Celastraceae: Salaciodeae) in peninsular India. Webbia. Journal of Plant Taxonomy and Geography76(2):167-193. doi:10.36253/ jopt-10390

Received: January 28, 2021

Accepted: April 7, 2021

Published: September 7, 2021

Copyright: (C) 2021 Mayur Dhondiram Nandikar. This is an open access, peer-reviewed article published by Firenze University Press (http://www. fupress.com/webbia) and distributed under the terms of the Creative Commons Attribution License, which permits unrestricted use, distribution, and reproduction in any medium, provided the original author and source are credited.

Data Availability Statement: All relevant data are within the paper and its Supporting Information files.

Competing Interests: The Author(s) declare(s) no conflict of interest.

Editor: Riccardo M. Baldini

ORCID

MDN: https://orcid.org/0000-00018626-3669

\section{The genus Salacia (Celastraceae: Salaciodeae) in peninsular India}

\author{
Mayur Dhondiram Nandikar \\ Naoroji Godrej Centre for Plant Research (NGCPR), 431 Lawkim Campus, Shirwal, Sata- \\ ra, Maharashtra, 412801, India \\ Email: nandikar@godrej.com; mnandikar@gmail.com
}

\begin{abstract}
The genus Salacia (Celastraceae: Salaciodeae) in peninsular India is revised, wherein ten species are recognised. Eight species are endemic to the region. Salacia macrophylla and S. reticulata are excluded from the revision as occurrence of both the species is doubtful in mainland India. Salacia macrosperma exhibits great variation in leaf shape, flower number, pedicel length and fruit texture, hence the morphologically similar species $S$. beddomei and S. vellaniana are relegated to its synonymy. Descriptions, photographs, and pen and ink as well as colour drawings of all recognised Salacia species are provided with an artificial key, updated nomenclature, and synonymy. Distribution and IUCN Red list categories and criteria are also discussed. Seven binomials, S. fruticosa, S. macrophylla, S. macrosperma, S. oblonga, S. ovalis, Tontelea prinoides and Johnia coromadeliana are lectotypified.
\end{abstract}

Keywords: Hippocrateaceae, India, Salacia, taxonomy, Western Ghats.

\section{INTRODUCTION}

Celastraceae consists of 96 genera and about 1300 species (Mabberley 2017), distributed in tropical, subtropical and temperate parts of the world. In India, the family (including Hippocrateaceae) is represented by 15 genera and about 114 species (Ramamurthy and Naithani 2000). The classification of Celastraceae has undergone several revisions based on molecular evidence and it is now recognized with four subfamilies (Celastroideae, Stackhousioideae, Hippocrateoideae and Salacioideae). Subfamily Salacioideae consists of six genera and most of the species within this subfamily are lianas. Cheiloclinium Miers, Peritassa Miers and Tontelea Miers are confined to South America, Salacighia Loes. and Thyrosalacia Loes. are native to tropical Africa, whereas one of the largest genera, Salacia L., is distributed in both the Old and New World tropics (Simmons 2004). Salacia is perhaps best known as the source of salacinol, an antidiabetic compound (Yoshikawa et al. 1997).

Among the members of Celastraceae from India, the species of Salacia resembles those of Loeseneriella A.C. Sm. and Reissantia N. Hallé, but differs by their axillary or ramiflorous fascicles, thyrsiform or paniculate cymes, intra-staminal fleshy, annular-pulvinate or flattened disc (Figure 1A), 2 or 3 

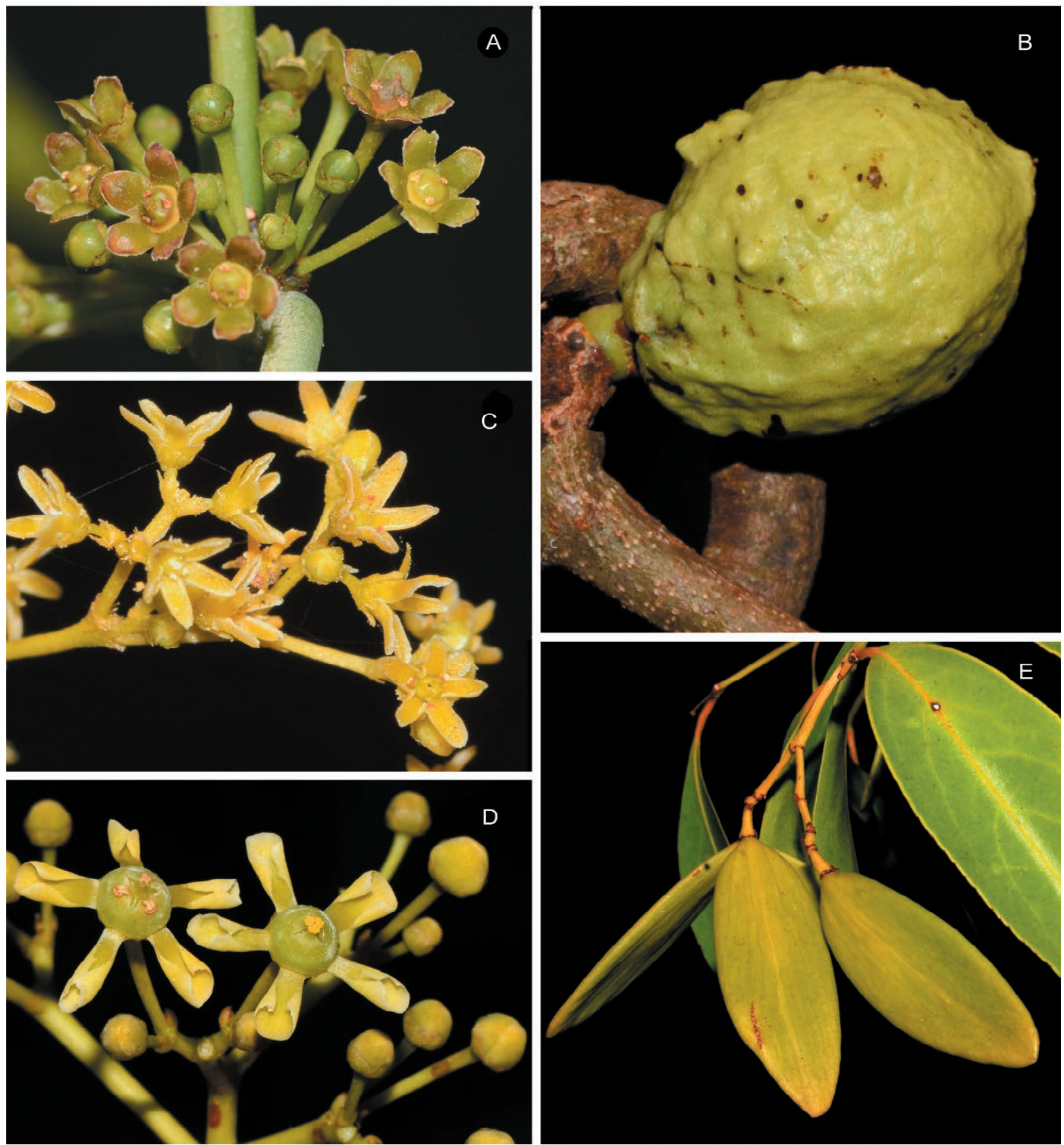

Figure 1. Inflorescences and fruits in different genera of Indian Celastraceae. (A) Salacia macrosperma Wight: axillary fascicle and oblong petals. (B) S. gambleana: indehiscent berry-like or drupaceous fruit. (C) Reissantia grahamii: branched cymes with inconspicuous floral disc. (D) Loeseneriella arnottiana: branched cymes with conspicuous floral disc and involute petals. (E) Reissantia indica: dehiscent capsules.

inserted stamens from the pistil base, 2- or 3-celled, 1-8 ovulate ovary, drupaceous or baccate; mucilaginous (pulpy) or dry meso or endocarp, 1-8-seeded, indehiscent fruits (Figure 1B). Reissantia has entire-margined petals and an inconspicuous disc (Figure 1C), whereas Loeseneriella has involute petals and a conspicuous disc (Figure 1D). Moreover, both these latter genera possess axillary, dichotomously branched cymes, with or without 
supplementary branchlets in dichotomy, capsular, dehiscent fruit, and winged seeds (Figure 1E). Historically these three genera were often placed in Hippocrateaceae along with Hippocratea L. In more recent classifications, however, Hippocrateoideae and Salacioideae are included in Celastraceae as monophyletic groups representing different lineages. Fruits of Hippocrateoideae are dehiscent mericarps with winged seeds, whereas those of Salacioideae are indehiscent and drupaceous (Robson 1965; Ding Hou 1964; Robson et al. 1994; Simmons et al. 2001; Coughenour et al. 2010, 2011).

The genus Salacia consists of about 200 species worldwide (Mabberley 2017). The group is most diverse in tropical Africa with approximately 90 species (Hallé 1962); remaining taxa are distributed in southeast Asia with nearly 30 species (Ding Hou 1964; Pelser et al. 2016), and neotropics with 33 species (Smith 1940; Hedin 1999; Lombardi 2014). Ramamurthy and Naithani (2000) recognized 21 species of Salacia in India, 11 of which are endemic to the country. Subsequently three further species and one variety have been described from peninsular India (Udayan et al. 2012, 2013, 2014; Sujana et al. 2015, Page and Nandikar 2020).

Among the Indian species, Salacia floribunda Wight, S. jenkinsii Kurz and S. khasiana Purkay. are known from North East India, whereas S. majumdarii (Chakrab. \& M.Gangop.) B.D. Naithani, S. platyphylla Kurz and S. tortuosa Griff. occurs on the Andaman and Nicobar Islands. Salacia grandiflora Kurz, S. salacioides (Roxb.) R.S.Rao \& Hemadri and S. verrucosa Wight are common to North East India as well as the Andaman and Nicobar Islands. S. chinensis L. occurs throughout peninsular India and the Andaman Islands, whereas $S$. oblonga Wight \& Arn. is restricted to the Western Ghats of India and Sri Lanka.

Salacia is more diverse in peninsular India than other parts of the country. Of the ten species recorded so far, eight are endemic to this part of the country: S. agasthiamalana Udayan, Yohannan \& Pradeep, S. brunoniana Wight \& Arn., S. fruticosa Heyne ex M.A.Lawson, S. galmbleana Whiting \& Kaul, S. macrosperma Wight, S. malabarica Gamble, S. megacarpa N.V.Page \& Nandikar and S. wayanadica Sujana, Nagaraju, M.K.R.Narayanan \& Anil Kumar. Salacia megacarpa is the only species that has large, ridged, green mature fruit with a dry endocarp. Salacia agasthiamala$n a$ is unique in having small, elliptic, obovate leaves and fruits with a thin epicarp. Salacia vellaniana Udayan, Yohannan \& Pradeep, wrongly synonymized under $S$. gambleana by Sasidharan \& Anaz (2015), is considered conspecific to $S$. macrosperma. In $S$. macrosperma, the fruits have been described unclearly. In the protologue, fruits were given as irregularly ovate, and in illustration fruit vary from globose to obovoid to ellipsoid, smooth (Wight 1845). The original material (housed at K) has broadly ellipsoid-oblong fruit. Nevertheless, most collections of S. macrosperma from peninsular India also bears globose to oblong fruits with a smooth to sparsely tuberculate exocarp. Hence, taxonomic delimitation of $S$. macrosperma from its congeneric S. beddomei Gamble and $S$. brunoniana is often difficult. Incidentally, both the latter species were described without fruit. In the case of $S$. beddomei, it was found to be conspecific with $S$. macrosperma due to similarities in leaf shape, size, flowers. On the other hand, S. brunoniana is retained as distinct species, as it differs from $S$. macrosperma by having serrate leaves, and few-flowered fascicles.

The claimed occurrence of $S$. macrophylla and $S$. reticulata in peninsular India by Ramamurthy and Naithani (2000) is based on misidentifications. The former was identified based on the work of Ding Hou (1964), which was based on Dalzell's collection from Konkan. The present author had an opportunity to scrutinize Dalzell's collection of S. oblonga at $\mathrm{K}$ which is erroneously determined by Ding Hou as S. macrophylla. Similarly, S. reticulata Wight (1840) does not occur in India. It was based on a collection of Colonel Walker from Ceylon (Sri Lanka). In the protologue, Wight was uncertain about its distribution in India. Subsequently, Gamble (1918) incorrectly included this species based on a collection of Rao (87440 at CAL), and misinterpreted the type specimen (C.P. 2720 at CAL) of the Sri Lankan endemic $S$. diandra Thwaites as $S$. reticulata (see Udayan and Pradeep 2012).

\section{Brief taxonomic history of Salacia in India}

'Courondi' was the earliest recorded vernacular name for a species of Salacia, used by Van Rheede (1683) in his Hortus Malabaricus and later identified as Salacia chinensis (Ding Hou 1964, Nicolson and Suresh 1986), although it should rather be $S$. fruticosa (see the note under $S$. fruticosa in the present contribution). The name 'Courondi' was validated as a new genus Courandi Adanson (1763), but later rejected against the earlier Linnaean Salacia (Dandy 1969, Nicolson and Suresh 1986). Roxburgh (1820) in his Hortus Bengalensis, used the generic name Johnia for members of Salacia and subsequently applied it to J. salacioides Roxb. (= Salacia salacioides) and J. coromandeliana Roxb. (= Salacia chinensis L.) in his Flora indica.

Wight and Arnott (1834) described three Salacia species: S. brunoniana, S. oblonga and S. oppositifolia Wight \& Arn., of which S. oblonga is distributed in 
peninsular India and Sri Lanka, whereas S. brunoniana is endemic to peninsular India. Salacia oppositifolia and $S$. oblonga are now found to be conspecific. Wight $(1840,1845)$ described four new species, S. floribunda Wight, S. reticulata, S. verrucosa and S. macrosperma Wight. Bentham and Hooker (1862) in Genera Plantarum reduced Hippocrateaceae to the tribes Celastreae and Hippocrateae. Kurz $(1872,1875)$ described S. grandiflora Kurz from Myanmar. S. jenkinsii Kurz and S. platyphylla Kurz were described from Assam and the Andaman and Nicobar Islands, respectively.

Lawson (1875) in the treatment of Flora of British India, placed Salacia in the tribe Hippocrateae and order Celastrineae. He recognised 20 species in the genus, with Salacia latifolia Wall. ex M.A.Lawson (=S. chinensis) considered as doubtful species, and S. fruticosa a new species based on Heyne's collection. Gamble (1916) described three species, S. beddomei, S. malabarica and S. talbotii Gamble from southern peninsular India. Purkayastha (1938) added S. khasiana from Assam.

Ding Hou (1964) revised the Malaysian Celastraceae and recorded 29 species, of which five species share a distribution with Andaman and Nicobar archipelago. With the addition of $S$. korthalsiana Miquel and S. tortuosa (Lakshminarasimhan and Srivastava 1993, Lakshminarasimhan and Ray 1994), the Andaman and Nicobar Islands now harbours seven Salacia species: $S$. platyphylla, S. nicobarica, S. korthalsiana, S. chinensis, S. reticulata, $S$. salacioides and $S$. verrucosa, with the first two endemic to these islands.

\section{Biogeography and comparative morphology of Indian Salacia}

Salacia chinensis, S. macrosperma and S. oblon$g a$ occur on the plateaus, along forest fringes and in undergrowth. Salacia chinensis also occurs in dryland, and riverine forests of peninsular India. Salacia fruticosa and S. malabarica grow along forest margins and in coastal scrubland forest. Salacia gambleana occurs along forest margins and often shares the habitat with S. macrosperma. Salacia agasthiamalana and S. wayanadica are known only from the type localities. The former grows on the slopes in evergreen grassland at an altitude of $900-1100 \mathrm{~m}$ and is narrowly distributed in Agasthiamala Biosphere Reserve and adjoining areas, whereas the latter has a sporadic distribution along the forest margin in Wayanad District, Kerala. Similarly, $S$. megacarpa is known from wet evergreen forests bordering Kerala and Karnataka. Except for its type collection from Nilgiris (Tamil Nadu), S. brunoniana is known only from evergreen forests of Palode, Thiruvananthapuram, Kerala.
Salacia agasthiamalana is a shrub or small shrub, whereas $S$. chinensis is often recorded as small tree to scandent shrub. All the other Salacia species grows either as scandent shrubs or lianas, straggling over small trees. Branchlets are usually grey to dark brown or black, smooth to lenticellate; rusty, abruptly yellowstriated in S. oblonga (Figure 2A-C). Leaf shape (Figure 2D) varies from obovate, oblanceolate, ovate to ellipticoblong. S. agasthiamalana has small, elliptic to obovate leaves in comparison with the rest of the species in peninsular India. S. gambleana has distinct oblanceolate leaves. The leaf margin also varies from entire to serrate, cuneate, sinuate, undulate, whereas the leaf apex varies from acute to acuminate or obtuse. The midrib and lateral nerves are usually conspicuous, rarely inconspicuous as in S. macrosperma, with the lateral nerves varying from 6 to 12 pairs. Some of the African species are characterised by resinous or latex threads, particularly when breaking of leaves and petiole (Robson et al. 1994), which is absent in peninsular Indian Salacia. The inflorescence usually consists of solitary to many flowered ramiflorous and axillary fascicles (Figure 2E), whereas pedunculate cymes (Figure $2 \mathrm{~F}$ ) are characteristic of $S$. fruticosa, S. oblonga and S. megacarpa.

The flowers (Figure $3 \mathrm{~A}-\mathrm{G}$ ) are moderately sized (7-15 $\mathrm{mm}$ in diam.), usually salver-shaped (urceolate in S. oblonga); pedicel length varies from 0.3 (S. oblonga) to 2 (S. malabarica) $\mathrm{cm}$; bracts are often conspicuous and sepaloid; buds are usually globose, with calyptriform sepals, ovoid to conical in S. chinensis. The sepals are usually fused at the base, with the sepal lobes (Figure $4 \mathrm{~A}-\mathrm{H}$ ) laciniate to fimbriate in S. agasthiamalana, $S$. macrosperma, and S. gambleana, glabrous or marginally or apically erose in S. megacarpa, S. chinensis, S. oblonga and S. brunoniana, sparsely ciliate in S. malabarica, apically bearded in $S$. wayanadica, inconspicuous, undulate, or crisp, white-margined in S. fruticosa. The petals are primarily green to pale green, elliptic to oblong, often with hyaline margins, revolute, obovate to oblanceolate in S. chinensis and S. megacarpa, orbicular and orange to ochreous in $S$. fruticosa. Disc shape, size and colour varies with flower maturity, oblate and green to pale green in S. chinensis, otherwise conical or lenticular, entire, or faintly lobed, green with orange shades or ochreous. There are three stamens, the filaments often becoming reflexed or revolute; anthers bi-thecate, globose to ellipsoid, thecae confluent by oblique slits in $S$. megacarpa (Figure 5A), longitudinal slits (Figure 5B) in S. fruticosa and S. oblonga. Ovoid, yellow to ochrayellow anthers and thecae confluent by transverse slits (Figure 5C) are most common in the remaining species of peninsular India; pollen simple, tricolporate; ovary 

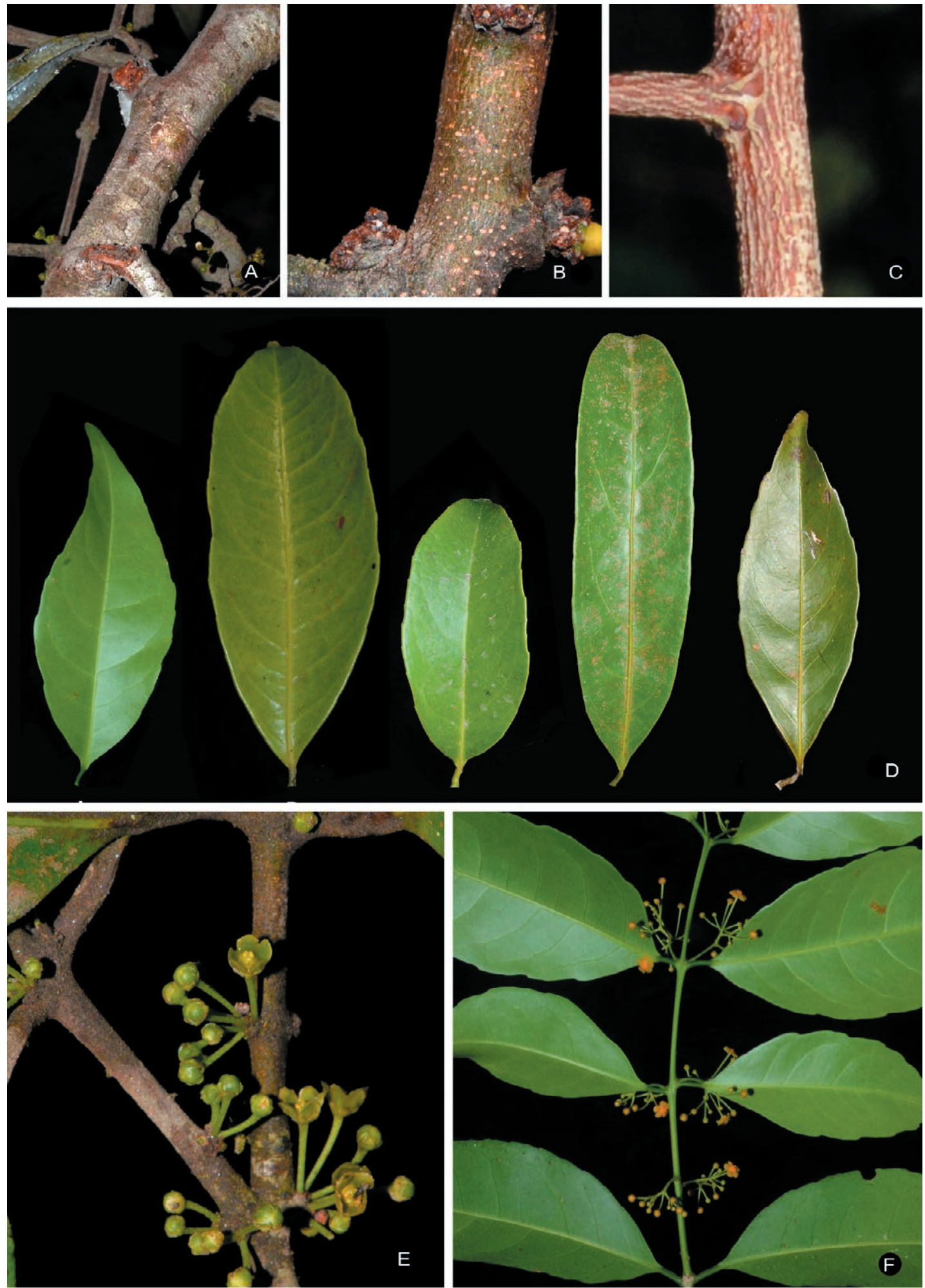

Figure 2. Lateral branches (A-C) and inflorescences (E-F) in species of Salacia. (A) Salacia wayanadica: smooth surfaces and hooked branches. (B) S. macrosperma: reticulate branches. (C) S. oblonga: irregularly corrugated. (D) Leaves (S. fruticosa: ovate to lanceolate, faintly crenate, acuminate; S. macrosperma: large, broadly elliptic, entire to faintly serrate, acute-obtuse; S. chinensis: small, elliptic, serrate; S. oblonga: large, oblong, entire, acute-obtuse; S. brunoniana: lanceolate, serrate-crenate, acuminate. (E) S. wayanadica: axillary and ramiflorous, 2-8-flowered fascicle. (F) S. fruticosa: axillary and ramiflorous, dichotomously branched, few-flowered cyme. 

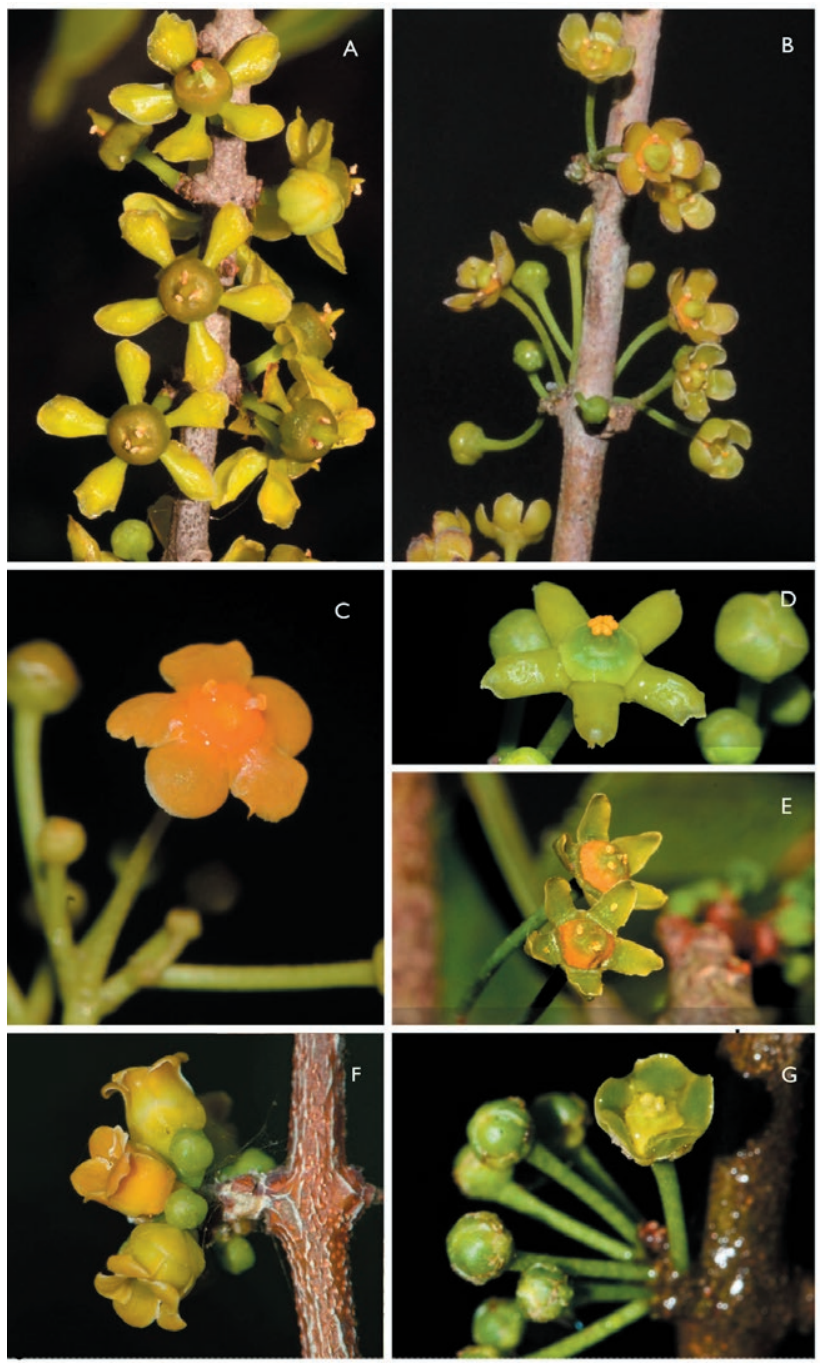

Figure 3. Flowers in species of Salacia. (A) Salacia chinensis: oblanceolate, pale green petals. (B) S. brunoniana: elliptic-oblong, ochreous petals with orange disc. (C) S. fruticosa: globose or orbicular, orange to ochreous petals, flattened, orange disc. (D) S. megacarpa: oblong, spreading, green petals with oblique anther cells. (E) $S$. malabarica: ovate to elliptic petals with orange disc. (F) S. oblonga: axillary, shortly pedunculate cymes, and urceolate flowers. (G). S. wayanadica, broadly elliptic-oblong petals.

immersed or embedded in the disc. Fruits (Figures 6, 7) usually a globose or ellipsoid berry, with pulpy mesoand endocarp and 1- (S. chinensis) to 8-seeded (dry indehiscent and prolate in $S$. megacarpa); exocarp smooth, or keeled, or verrucose, or tuberculate, thin (S. chinensis), otherwise leathery or tough; seeds ellipsoid to ovoid or oblate to obconical, usually dorsiventrally compressed, with surface smooth or reticulate.

\section{MATERIALS AND METHODS}

Multiple accessions of Salacia species were collected from different states of peninsular India. Photographs of habit, inflorescences, flowers, fruit, and seeds were taken using Nikon D7000 and Canon 700D digital cameras with different optics. Microscopic study was carried out with a Leica Stereo Microscope (DM500). Herbarium specimens were prepared based on the methods proposed by Forman and Bridson (1992). The processed and identified specimens were deposited in Naoroji Godrej Centre for Plant Research (NGCPR) Herbarium and duplicates presented to CAL and BSI. Existing herbarium specimens from the study area (peninsular India) were consulted at BSI, CAL, CALI, CMPR, FRLH, $\mathrm{K}, \mathrm{MH}, \mathrm{PBL}, \mathrm{RHT}$ and TBGRI. Images of herbarium specimens were virtually consulted using JSTOR Global Plants (https://plants.jstor.org/) and herbaria webpages from A, BISH, BM, E, F, FR, G, GH, NY, P, U and US. The provisions of the ICN (Turland et al. 2018) was applied to update the nomenclature and for purposes of typification.

Descriptions were based on observation of morphology, study of type specimens, the protologue and herbarium specimens, whereas the descriptive terminology follows Ding Hou (1964) and Robson et al. (1994). The colour illustrations were prepared by the author from photographs, microscope images and herbarium specimens using water colour on hot press paper and processed in Adobe Photoshop 7.0. Conservation status was assigned, and threat assessment carried out for global level using IUCN Red list categories and criteria (IUCN 2019) based on population size, threats, and the estimation of the geographical range size (EOO and AOO), by using GeoCat (Moat 2007). The population trend and size data were produced from field observations, available literature, previous assessments and from information on herbarium specimen labels. Distribution maps were prepared for endemic species using DIVA GIS 7.5, with herbarium and field data being used to plot the distribution points.

\section{TAXONOMIC TREATMENT}

Key to the species of Salacia in peninsular India

1. Inflorescence pedunculate cymes; flowers subsessile to distinctly pedicellate; anther cells (thecae) ovoid, confluent by longitudinal or oblique slits; fruit smooth (sparsely tuberculate in S. oblonga).

1. Inflorescence sessile, fascicles or umbelliferous cymes; flowers distinctly pedicellate; anther cells (thecae) globose to 


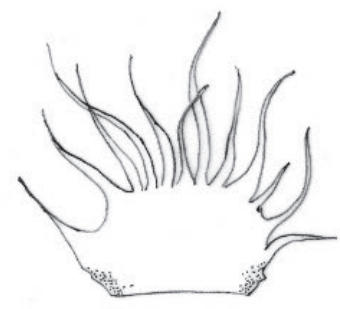

A

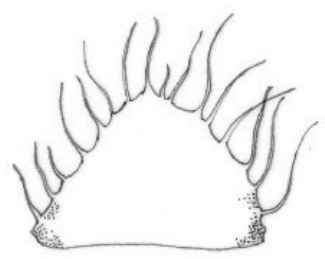

E

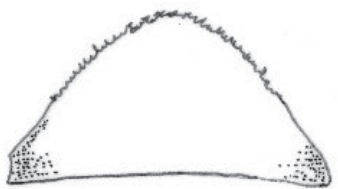

B

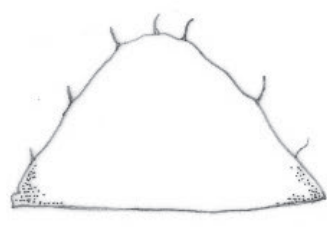

$\mathrm{F}$

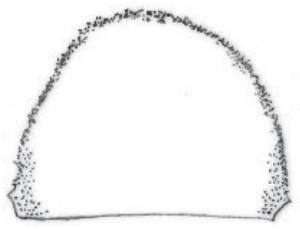

C

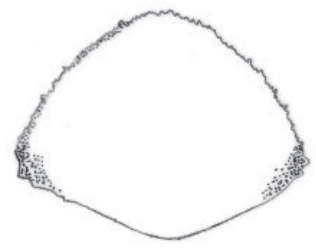

G

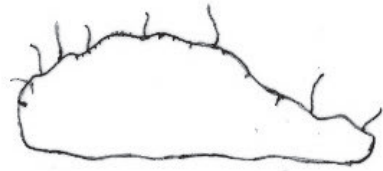

D

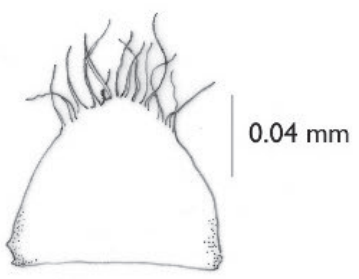

$\mathrm{H}$

Figure 4. Sepal lobes in species of Salacia. (A) Salacia agasthiamalana (B) S. brunoniana (C) S. chinensis, S. megacarpa (D) S. fruticosa (E) S. macrosperma, S. gambleana (F) S. malabarica (G) S. oblonga (H) S. wayanadica

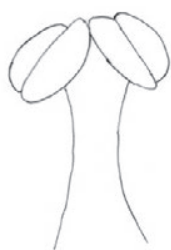

A

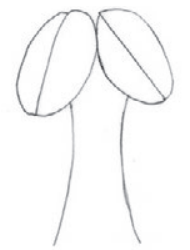

B

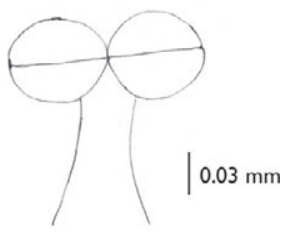

C
Figure 5. Stamens in species of Salacia. (A) Oblique anther cell (theca): Salacia megacarpa (B) longitudinal anther cell: S. oblonga (C) Globose, transverse anther cell: common in rest of Salacia in peninsular India.

ellipsoid, confluent by transverse slits; fruits smooth or smooth to tuberculate or verrucose

2. Young branchlets rusty, yellow-striated; leaves ellipticoblong or oblanceolate, drying yellow; flowers urceolate; fruit globose to ovoid, smooth to faintly tuberculate.

S. oblonga

2. Young branchlets grey to dark brown, smooth; leaves ovatelanceolate or broadly ovate or elliptic-lanceolate, drying green or black; flowers with spreading petals; fruit globose to prolate to broadly ellipsoid, smooth, or ridged .... 3

3. Sepal lobes undulate, crisped, faintly ciliate; petals orbicular, ochreous; fruit globose, $2.5-3.5 \times 2-3 \mathrm{~cm}$, smooth with 2 or 3 -seeded mucilaginous (pulpy) endocarp..... S. fruticosa

3. Sepal lobes entire, smooth to erose; petals elliptic-oblong or obovate, grey-green to yellowish green; fruit prolate to broadly ellipsoid, 7-10 $\times 4-6 \mathrm{~cm}$, ridged or keeled with 5-seeded dry endocarp

S. megacarpa

4. Flower buds ovoid or conical; petals obovate to oblanceolate, margin revolute; disc oblate; berries 1-seeded, exocarp smooth, thin.

S. chinensis

4. Flower buds globose; petals elliptic, ovate to oblong, margin undulate or crisped; disc conical or lenticular; berries 1-8-seeded, exocarp smooth to tuberculate, or smooth, or verrucose, leathery. ... 5

5. Erect shrubs; petiole 4-7 $\mathrm{mm}$ long; berries smooth.

S. agasthiamalana

5. Scandent shrubs, or lianas; petiole $8-20 \mathrm{~mm}$ long; berries smooth, or smooth to tuberculate or verrucose.

6. Pedicel 1.5-2 (-2.5) cm long; sepals entire to sparsely ciliate S. malabarica

6. Pedicel $0.8-1.2 \mathrm{~cm}$ long; sepals bearded, erose to laciniate or fimbriate 7

7. Leaf apex obtuse, margin entire; sepals bearded at apex with long cilia; petals oblong, pale green, tinged with orange

S. wayanadica

7. Leaf apex acute to acuminate, margin entire to undulate, or sinuate to crenate, or serrulate; sepals smooth to erose or laciniate; petals ovate to broadly elliptic, green to pale green ... 8

8. Leaves sinuate to cuneate, $4.6-7.5 \times 2.7-4 \mathrm{~cm}$; sepals inconspicuous, faintly erose at apex; petals oblong.

S. brunoniana 

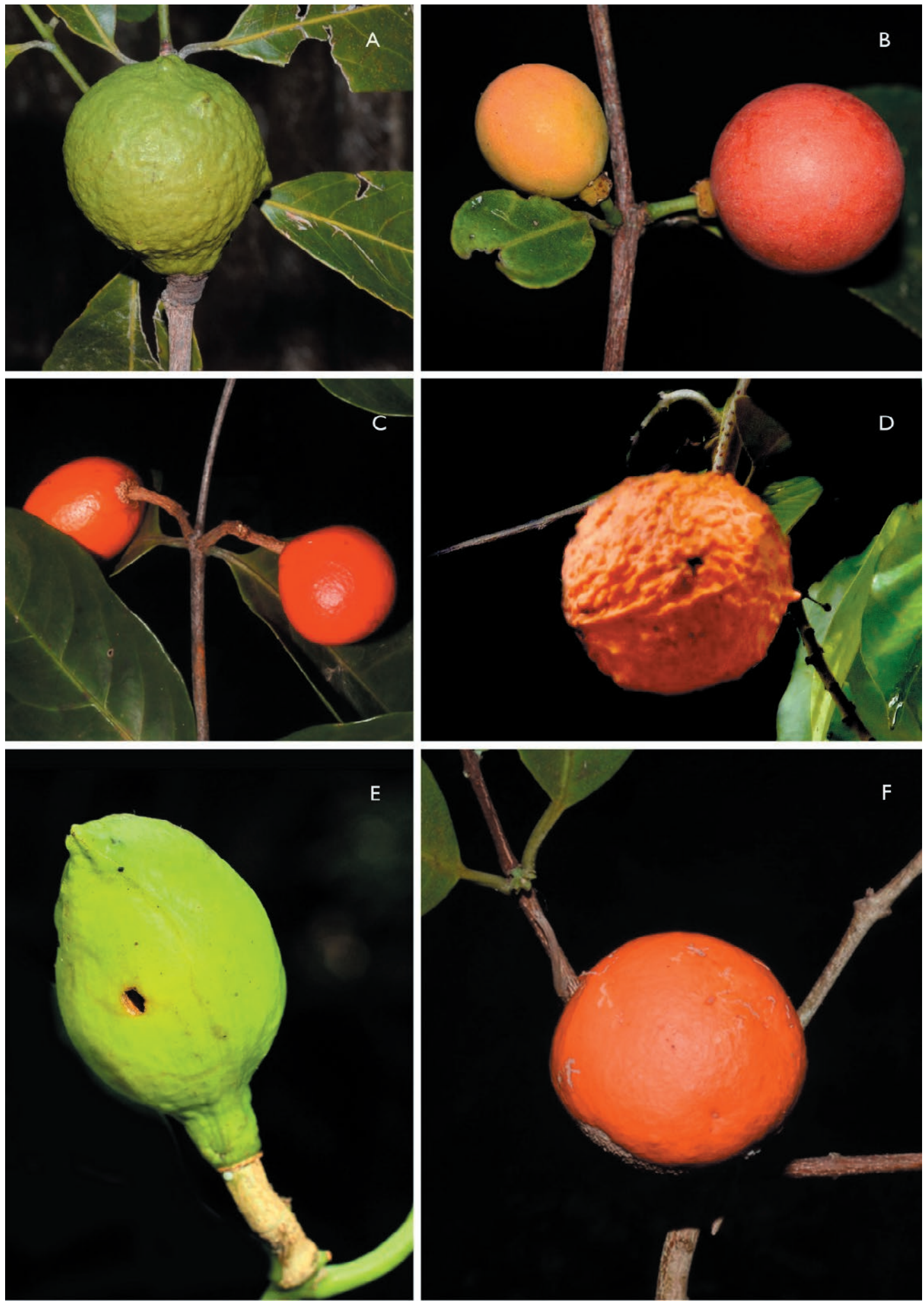

Figure 6. Fruits in species of Salacia. (A) S. brunoniana: immature, obovoid fruit with faintly tuberculate exocarp. (B) S. chinensis: smooth, globose, thin with pale orange exocarp with persistent disc base. (C) S. fruticosa: smooth, globose to ovoid, leathery, orange exocarp with distinct stalk. (D) S. malabarica: reticulate-verrucose, globose, orange exocarp. (E) S. megacarpa: immature, ovoid, faintly ridged fruit with distinct stalk. (F) S. oblonga: smooth, globose, orange exocarp. 

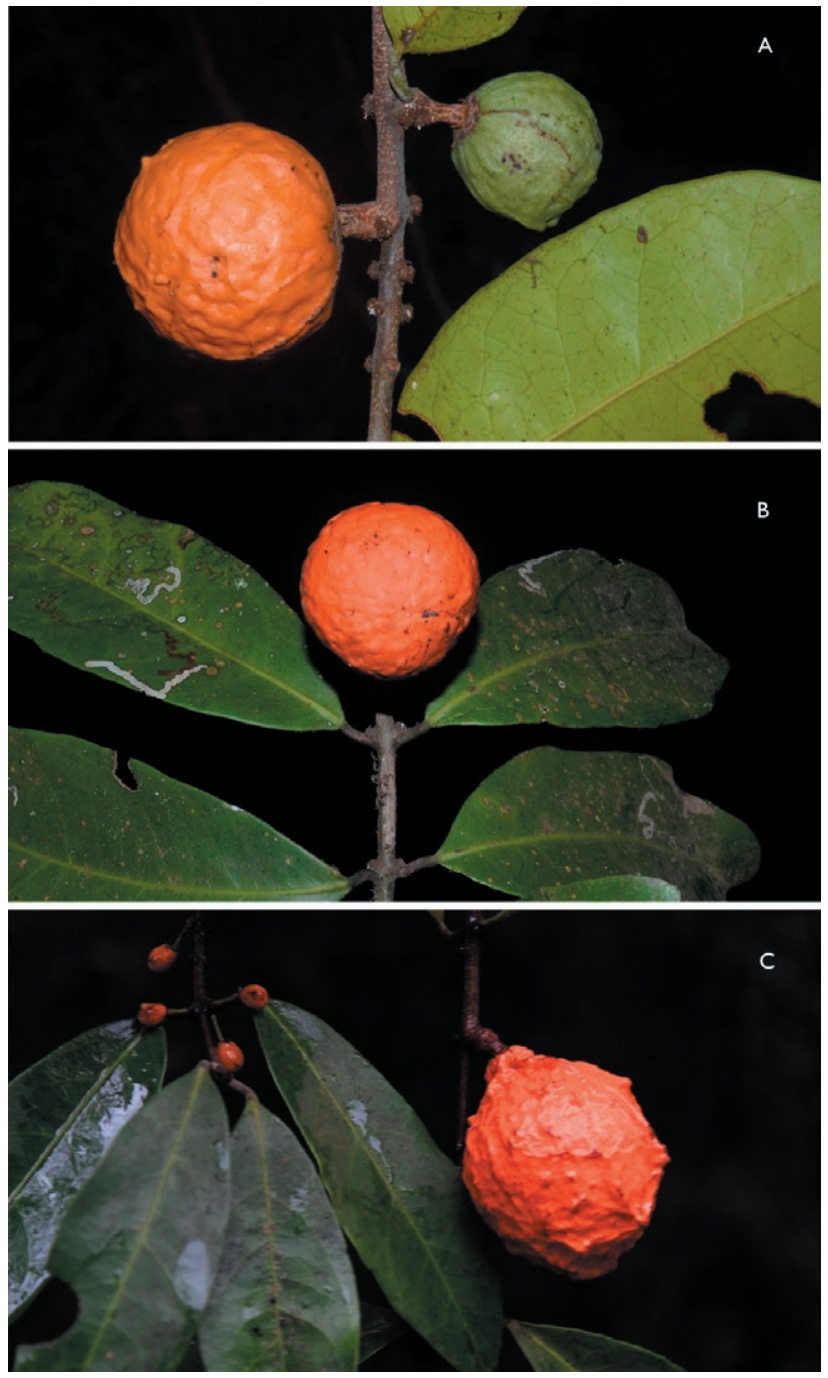

Figure 7. Exocarp variations from smooth to tuberculate $(A-C)$ in S. macrosperma.

8. Leaves entire, undulate, serrate, $6-15 \times 3-7 \mathrm{~cm}$; sepals characteristically laciniate, or fringed; petals ovate to elliptic.. 9

9. Leaves usually oblanceolate, rarely elliptic-oblong, lateral nerves 6-8 pairs; berries smooth to verrucose or verrucose-tuberculate.

S. gambleana

9. Leaves elliptic-oblong or obovate-elliptic, lateral nerves 6-12 pairs; berries smooth to tuberculate

S. macrosperma

Salacia L. Mant., Pl. 2: 159. 1771. nom. cons.

Type: Salacia chinensis L.

Courondi Adans., Fam. Pl. 2: 446. 1763. nom. rej.
Type: 'Courondi' Rheede

Christmannia Dennst., Schlüssel Hortus Malab. 30: 1818.

Type: Christmannia courondi Dennst.

Johnia Roxb., Fl. Ind. 1: 172. 1820.

Type: Johnia salacioides Roxb.

Salacicratea Loes., Nova Guinea 8: 281. 1910.

Type: Salacicratea papuana Loes.

\section{Description}

Perennial scandent shrubs, lianas or woody climbers, rarely small trees or shrubs, distal branches scandent, glabrous throughout or essentially so; branchlets terete, often hooked, lenticellate, irregularly corrugated, swollen or somewhat flattened at nodes. Leaves opposite or sub-opposite, decussate, stipules inconspicuous or absent; petiole 4-15 $\mathrm{mm}$; lamina ovate-elliptic, ellipticlanceolate, or oblanceolate or oblong-lanceolate; margin crenate, serrate, or entire; lateral nerves 5-12, anastomosing. Inflorescences axillary or ramiflorous few to many flowered, tuberculate fascicle or branched cymes; pedicels 5-20 mm long; sepals 5(4) lobed, broadly ovate to orbicular, margin laciniate, or fringed or entire or erose or ciliate; petals 5 obovate to oblanceolate, elliptic to oblong, or ovate to elliptic-lanceolate, or orbicular or oblanceolate, often revolute; disk intra-staminal, fleshy, annular-pulvinate, sometimes conical or flattened, rarely cupular; stamens 3 , inserted on disk, free or adnate with pistil, usually reflexed at anthesis, filaments subulate, broadened towards the base, anthers bilobed, thecae globose or ellipsoid or ovoid, confluent transversely or longitudinally or obliquely; ovary partly or completely immersed in the disk, 3 -celled (locular), ovules 1(2)-8 in each cell; style short, simple, stigma simple. Fruit baccate, globose, broadly ellipsoid, or prolate, 3-celled, indehiscent, exocarp smooth to tuberculate, or smooth, verrucose or ridged; seeds 1 to several, embedded or not in mucilaginous (pulpy) endocarp, variously shaped, cotyledons massive, free or united.

\section{General Distribution}

About 200 species distributed in tropical and subtropical Africa, America and Asia, with few species in the temperate parts of Asia and America (Ding Hou 1963; Mabberley 2017). India with 21 species, ten of which are reported from peninsular India.

1. Salacia agasthiamalana Udayan, Yohannan \& Pradeep in Edinburgh J. Bot. 69(2): 255. 2012. (Figure 8)

Type: India, Kerala, Thiruvananthapuram, Agasthiamala, 11 Apr. 2010 (flowering), Udayan \& Regy Yohan- 


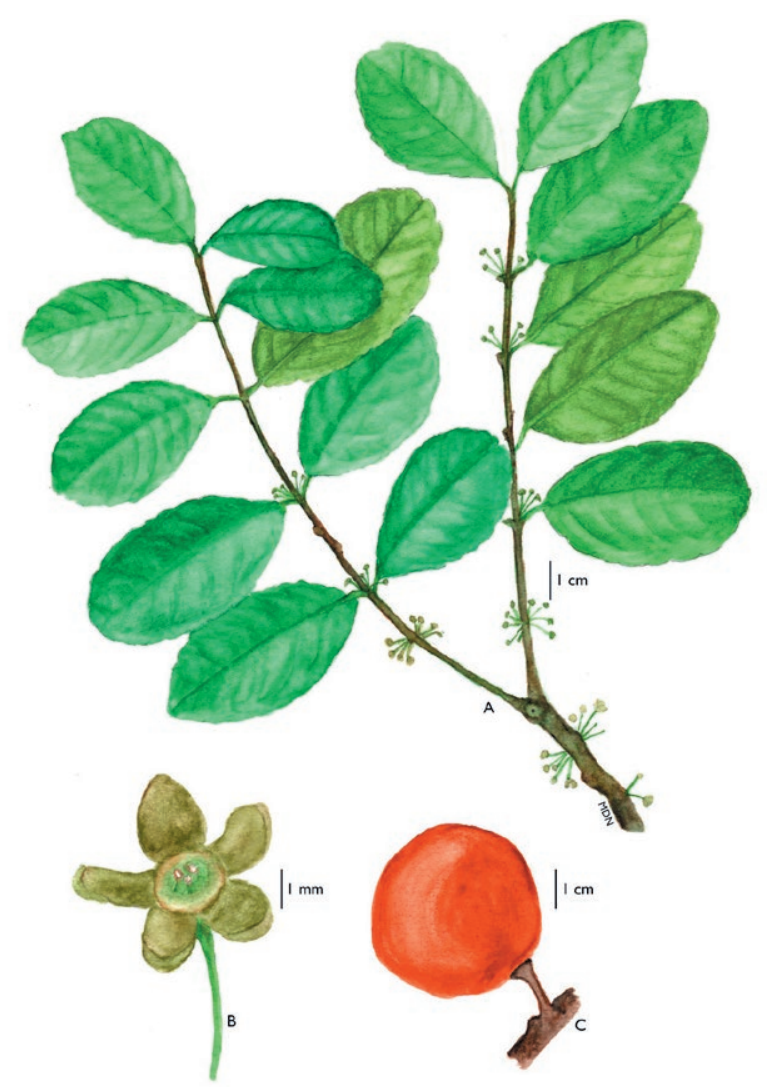

Figure 8. Salacia agasthiamalana (A) Flowering twig. (B) Flower. (C) Fruit.

nan S004 (holotype, MH n.v.; isotypes, K n.v., SKC n.v., SNCH n.v.).

\section{Description}

Erect shrubs; branchlets blackish lenticellate. Leaves opposite or subopposite, exstipulate; petiole 4-7 $\mathrm{mm}$ long; lamina broadly elliptic or obovate, $5-6 \times 2-4$ $\mathrm{cm}$, apex acute or rounded-retuse, base acute or cuneate, margin serrate to crenate, coriaceous. Inflorescences axillary or ramiflorous, tuberculate, 2-8-flowered fascicles; pedicel 5-11 mm long; sepals fused at base, lobes oblong, c. $1 \mathrm{~mm}$ long, margin fimbriate; petals broadly elliptic, c. $2 \times 1.5 \mathrm{~mm}$; disk conical, c. $0.5 \times$ $1 \mathrm{~mm}$, tapering into a short style; stamens 3 , rarely 4; anthers bi-lobed, globose, creamy-white with a brown tinge, thecae confluent transversely; filaments slightly spreading, broad at the base; ovary 3-celled, 1-ovulate; stigma simple, pale green. Fruits baccate, shortly stipitate, globose, exocarp smooth, leathery, 4-6 cm across, orange-red, 3-seeded; seeds slightly plano-convex, 1.5$2 \times 1-1.5 \mathrm{~cm}$.
Phenology

Flowers in March, fruits remain till June.

\section{Distribution and habitat}

Endemic to peninsular India, the species is known from two localities Pongalapara, Agasthiamalya, Thiruvananthapuram in Kerala and Peyaru to Panimalai, in Tirunelveli, Tamil Nadu (Figure 18). It grows on slopes of evergreen grassland at the altitudinal range of 9001100 m (Udayan et al. 2012; Rajasekar et al. 2018).

\section{IUCN status}

Both the known locations of Salacia agasthiamalana are adjoining, Pongalapara is a protected area (part of Agasthiamala Biosphere Reserve), where no threat has been recorded so far. No information is available for the population at Panimalai. Hence, due to insufficient data on the population size and threat the species is assessed here as Data Deficient (DD).

\section{Specimens examined}

INDIA. Kerala, Thiruvananthapuram, Agasthiamala, Pongalapara, 11 Apr. 2011, Udayan \& Regy Yohannan $00 S 9(\mathrm{MH})$.

Notes

S. agasthiamalana is similar to $S$. macrosperma Wight in being cauliflorous, with 1- to many-flowered fascicles, but differs in having a bushy habit, blackish, lenticellate branchlets, obovate, small, obtuse leaves and globose, smooth fruits. It also resembles $S$. chinensis in habit, leaf size and shape, however, shortly petiolate, obtuse leaves, broadly elliptic petals, 3-seeded, 4-6 cm large fruits distinguish $S$. agasthiamalana.

Type material of $S$. agasthiamalana at the herbaria listed in the protologue could not be traced. The specimen at MH (Udayan \& Regy Yohannan 00S9), collected from the type locality, was used here to describe and illustrate the species (flower and fruits characters obtained from the protologue and Rajasekar et al. 2018).

2. Salacia brunoniana Wight \& Arn., Prodr. Fl. Ind. Orient. 1: 105. 1834. (Figure 9)

Type: India, Peninsular, s. d., Herb Wight 347 (lectotype, E! barcode E00174243), designated by Jadhav et al. (2016).

\section{Description}

Scandent shrubs or lianas up to $3(-5) \mathrm{m}$ high; branches looped, branchlets terete, glabrous. Leaves 


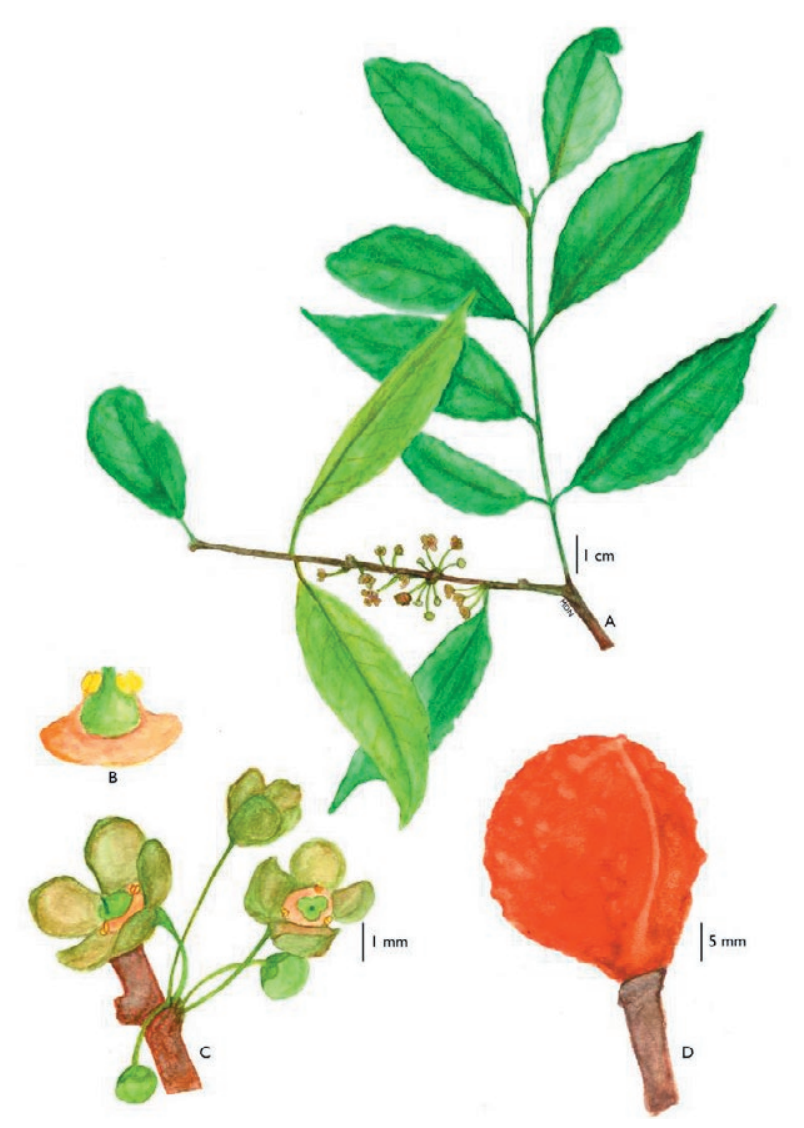

Figure 9. Salacia brunoniana (A) Flowering twig. (B) Disc. (C) Inflorescence. (D) Fruit.

opposite to sub-opposite or alternate, petiolate; petiole $0.8-1.5 \mathrm{~cm}$ long; lamina oblong or elliptic, 4.6-7.5 $\times 2.7-4 \mathrm{~cm}$, apex acuminate, base acute or cuneateattenuate, margin sinuate or crenate (rarely serrate), coriaceous, lateral nerves 5-8. Inflorescences axillary and ramiflorous, tuberculated, 2- to many-flowered fascicles; buds globose; pedicels $5-8 \mathrm{~mm}$ long; sepals fused at base, lobes ovate, inconspicuous, pale green, c. $1 \mathrm{~mm}$ long, adherent with the petals, faintly erose at apex; petals usually 5 , rarely 4 , oblong, c. $2.5 \times 2.2$ $\mathrm{mm}$, coriaceous, incurved, margin hyaline, yellowish green; disk flat or annular, fleshy, ochreous; stamens 3 , filaments $1 \mathrm{~mm}$ long, adnate to the disc or free, incurved, anthers bilobed, globose, pale ochreous, thecae confluent transversely; ovary oblate-globose, 2 - or 3 -celled, style c. $1 \mathrm{~mm}$ long, broad at base, tapering towards the apex, stigma inconspicuous. Fruit baccate, globose-obovoid, $2.5-4 \times 2-4 \mathrm{~cm}$, shortly stalked, exocarp tuberculate, orange-yellow at ripening, endocarp mucilaginous, 1-6-seeded; seeds ovoid to ellipsoid, $1-1.5 \times 1 \mathrm{~cm}$, brown.
Phenology

Flowers in January to March, fruiting remains till June.

Distribution and habitat

Endemic to the Western Ghats of Tamil Nadu and Kerala (Figure 18). Evergreen and fringing forest.

\section{Specimens examined}

INDIA. Tamil Nadu, Herb. Wight 365, Wall. Cat. no 4217E (K, K00069920); Nilgiris, 1884, M. A. Lawson 0326 (MH). Kerala, Vittalapacha, 18 Feb. 1992, A. Nazarudeen 13846 (TBGRI); ibid., 28 Feb. 2011, S. M. Shareef 70649 (TBGRI); ibid, 20 Mar. 2014, S. M. Shareef 79251 (TBGRI); Thiruvananthapuram, JNTBGRI, 13 Feb. 2014, S. M. Shareef 79235 (TBGRI); JNTBGRI, Palode, 16 Feb. 2017, D. C. Jadhav 1459 (NGCPR).

\section{IUCN status}

Salacia brunoniana is known from Tamil Nadu (Nilgiris), and Kerala (Thiruvananthapuram). At present there is no detailed information available on species distribution and population, therefore, considered here as Data Deficient (DD).

Notes

The distribution report of $S$. brunoniana in Maharashtra (Dalzell and Gibson 1861; Almeida 1996; Londhe 2000; Mishra and Singh 2001; Shareef and Kumar 2013) is an error. Dalzell's collection at K of S. brunoniana from Ram Ghat, Maharashtra is identified as $S$. chinensis. Nevertheless, I could not locate the collections by M.R. Almeida (2292: BNHS) and S.M. Almeida (5204: BLAT) (Almeida 1990) from Amboli, Sindhudurg Maharashtra. Similarly, its claimed occurrence in Odisha (Ramamurthy \& Naithani 2000), is based on it being mistaken for S. chinensis. Perhaps, they have referred to Panigrahi 23710 (CAL) labelled as S. brunoniana, which is an incomplete specimen of S. chinensis. Collections (Nazarudeen 13846, Shareef 70649: TBGRI) from Vittalapacha, Kerala well agrees with the protologue of $S$. brunoniana except in the number of flowers. S. brunoniana planted at JNTBGRI was originally brought from Vittalapacha, Kerala, which has distinctly sinuate, crenate, or serrate leaves, 2-8-flowered fascicle and broadly oblong-obovate petals. The species shares similarities in habit and flowering characters with S. macrosperma and S. gambleana, but these differs from S. brunoniana by characteristic laciniate, or fringed sepals and entire to serrate leaves. 
3. Salacia chinensis L., Mant. Pl. Altera 293: 1771. Tonsella chinensis (L.) Spreng., Syst. Veg., ed. 16, 1: 177. 1824. (Figure 10)

Type: China, Linn. Herb. No. 1066.1 (lectotype LINN! http://linnean-online.org/11292/), designated by Ding Hou (1963).

(=) Tontelea prinoides Willd., Neue Schriften Ges. Naturf. Freunde Berlin iv. 184: 1803.

Type: India, Orientalis, 1788, Klein s.n. ex herb. Willdenow (HAL! barcode HAL0118476), lectotype here designated.

(झ) Salacia prinoides (Willd.) DC., Prodr. [A. P. de Candolle] 1: 571. 1824.

(=) Johnia coromandeliana Roxb. in Fl. Ind., ed. Carey \& Wall., 1: 173. 1820.

Type: India, Roxburgh s.n. (BR! barcode BR6985653), lectotype here designated.

\section{Description}

Climbing shrubs or small trees up to $2(-4) \mathrm{m}$ high; much branched, branchlets angled, terete, often hooked, glabrous. Leaves opposite or subopposite, stipules deltoid; petiole $0.2-0.7 \mathrm{~cm}$ long; lamina oblong-lanceolate, elliptic to elliptic-lanceolate, obovate, or ovate-elliptic, $5-12 \times 2-5 \mathrm{~cm}$, apex acute to shortly acuminate, base cuneate or acute, margin entire, or faintly serrate-crenate, thin-coriaceous, lateral nerves 5-10 pairs. Inflorescences axillary and ramiflorous, tuberculate, 3-6-flowered fascicles; buds ovoid or conical; pedicel 5-7 mm long, glabrous; bract triangular or ovate, slightly erose; sepals fused at base, lobes ovate, sparsely erose; petals immersed from disk, obovate-oblanceolate, c. $3 \times 4 \mathrm{~mm}$, greenish yellow, margin revolute, often hyaline; disk annular-pulvinate, thick, oblate, often tapering into the short style; stamens 3, inserted on the disk, filament linear, subulate, $1 \mathrm{~mm}$ long, anthers bi-lobed, globose yellow, thecae confluent transversely; ovary 3 -celled, 2-ovulate, style short, conical, stigma simple. Fruits baccate, shortly stipitate, globose, $0.8-1 \mathrm{~cm}$ in diameter, minutely apiculate, raised on persistent oblate disk, exocarp thin, smooth, orange-red to reddish brown at ripening, endocarp hyaline, mucilaginous, 1-seeded; seed globose, 5-8 $\mathrm{mm}$ in diameter, rusty.

\section{Phenology}

Flowers from January, fruits may last till June.

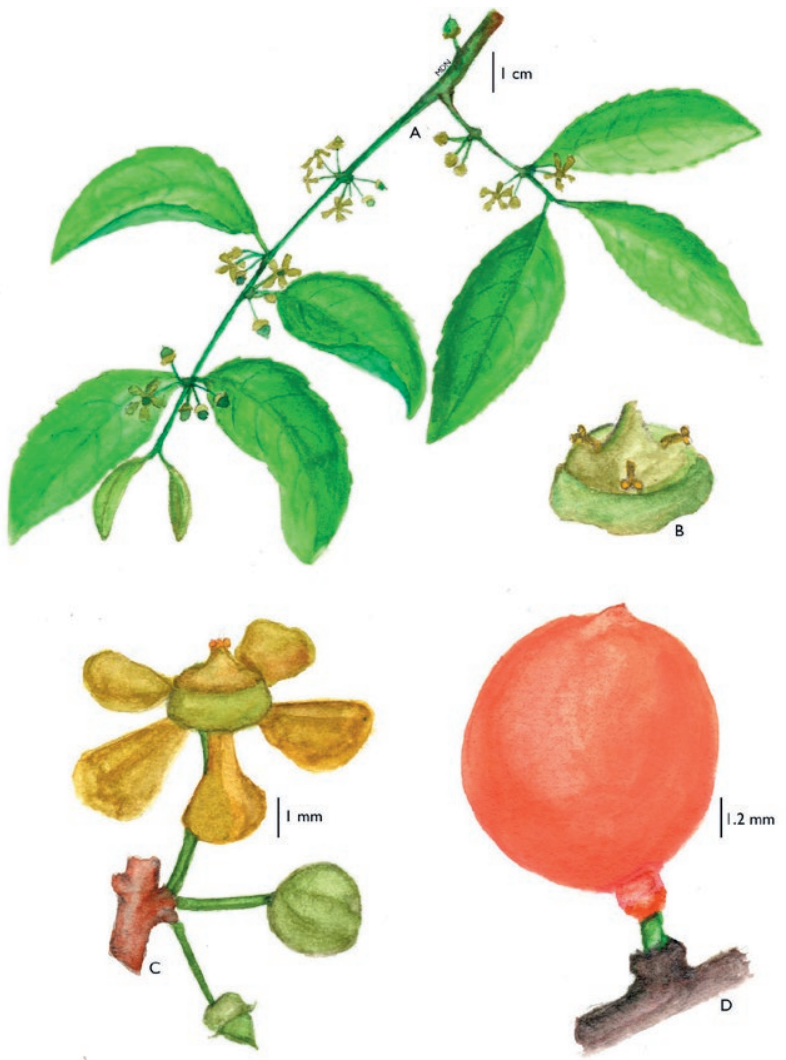

Figure 10. Salacia chinensis (A) Flowering twig. (B) Disc. (C) Inflorescence. (D) Fruit.

\section{Distribution and habitat}

Peninsular India, also in Andaman and Nicobar Islands, Assam, Odisha, and West Bengal; Sri Lanka and throughout southeast Asia. In open dryland forest, along the coastal lowland forest, as a small tree on open plateaus, in riverine forest and dense deciduous woodland.

\section{Vernacular names}

"madhu phal" (Bengali); "ekanaayakana beru" (Kannada); "cherukuranti" (Malayalam); "saptarangi", "nisul bondi" (Marathi); kadelanchi (Tamil); "saptachakra", "ekanayaka”, "pitika” (Sanskrit); "nerani” (Telugu).

\section{IUCN status}

As the species has a wide distribution range from Indo-Malaya to China, it is classified here as Least Concern (LC). Rare occurrence of S. chinensis in semievergreen forests of the Western Ghats (Southern India and Sri Lanka) mentioned by Kamat et al. (2020) is an error; its status as Endangered by Majid et al. (2016) is also an error. However, some individuals in the field (Shimoga, Amba, and Amboli) were observed with cut 
marks which may indicate its use for medicinal purposes among the local inhabitants. Salacia chinensis is the preferred species for the extraction of salacinol, an important antidiabetic compound. Often, it is found growing in areas of human habitation resulting on it being impacted by human activities such as road clearing, road widening, and overharvesting of its edible fruits. Often well grown plants have been uprooted from the wild, mainly for the roots as a source of salacinol (Patwardhan et al. 2014), which is non-renewable. However, considering its wide distribution, these threats do not yet pose a significant risk to the sustenance of the species in the wild. A detailed ecological assessment is required, throughout its range of distribution, to understand the population, regeneration, and threat.

\section{Specimens examined}

INDIA: Andaman and Nicobar Islands, Great Cocos Island, 1890, David Prain s. n. (CAL); Andaman, 1884, King s. n. (K, CAL); Baradaug, 10 Feb. 1915, C.E. Parkinson 290 (K); Ross Island, 29 Apr. C.E. Parkinson 517 (K); s. loc., 1884, King s. n. (K); ibid, King s. $n$. (K); South Nicobar, Navydera, 23 Oct. 1990, P.V. Sreekumar 15368 (PBL); Middle Andaman, Mayabunder, 03 May 1974, N. P. Balakrishnan 1306 (CAL); ibid, 29 Jan. 1959, K. Thothathri 9142 (CAL). Andhra Pradesh, Vizag, Palkonda hills, Mar. 1884, J.S. Gamble 14018 (K); ibid, J.S. Gamble 13898 (K). Assam, Khasi hills, s. d., Jenkins 37 (CAL); Abor Hills, 30 Jan. 1912, I. H. Burkill 36509 (CAL); Cachar, Jun. 1874, R. L. Keenan s. $n$. (K); Renjing, 28 Feb. 2012, I. H. Burkill 36739 (K). Karnataka, Karwar, 16 Feb. 1951, Joseph Fernandes 2145 (A, CAL); Mangalore, s. d., R. F. Hohenacker 405 (K); North Canara, 2 May 1995, W. A. Talbot 1197 (K); Sirsi, Jan. 1867, R. F. Hohenacker 769 (K); Banglore, FRLHT campus, 24 Aug. 2016, M. D. Nandikar 1458 (NGCPR); Shimoga, on the way to Honavar from Jog Fall, 21 Apr. 2017, D.C. Jadhav 1473; ibid, 2 Mar. 2018, D. C. Jadhav \& M.D. Nandikar 1481 (NGCPR). Kerala, Thiruvananthapuram, 16 Dec. 1893, M. A. Lawson 24 (K); Thiruvananthapuram, Palode, 5 Feb. 1996, Parmar 124 (K); Thiruvananthapuram, Pangappara, 10 May 1994, A. Nazarudeen 18778; JNTBGRI Medicinal Garden, 18 June 1996, A. E. Shanawas 29704 (TBGRI); ibid, 16 Feb. 2017, D. C. Jadhav 1463 (NGCPR). Maharashtra, Mumbai (Bombay), s. d., Dalzell s. n. (K); Ramghat, $s$. d., Dalzell s. n. (K); Sindhudurg, Amboli, 1 Dec. 1965, B.G. Kulkarni 107992 (BSI); Way to Ramghat, Amboli, 20 May 1965, R.D. Pataskar 105297 (BSI); ibid, 20 May 1965, R. D. Pataskar 105293 (BSI); Mangaon forest near Akeri, 23 June 1965, P. J. Cherian 102290 (BSI); Patgaon 5 miles from Ghotge, 27 Apr. 1966, B. G. Kulkar- ni 108516 (BSI); Khanyle 5 miles from Bhedshi, 20 Feb. 1966, M. Y. Ansari \& B. G. Kulkarni 108455 (BSI); Parwad wadi near Ghotge, 11 Feb. 1966, M. Y. Ansari \& B. G. Kulkarni, 107829 (BSI); Bhadgoan 12 miles from Ghotge, 28 Apr. 1966, B. G. Kulkarni 108538 (BSI); Parwadwadi, 2 miles from Ghotge, 26 Apr. 1966, B. G. Kulkarni, 108633 (BSI); Mahadevgad, Amboli, 30 Aug. 1967, B. G. Kulkarni 108666 (BSI); Bhadgoan near Kudal, 12 Feb. 1966, M. Y. Ansari 107866 (BSI); Songad, 36 km from Kankavli, 7 June 1970, B. G. Kulkarni 121096 (BSI); Belachi Ravi 24 km from Kankavli, 7 Oct. 1970, B.G. Kulkarni, 121528 (BSI); Mali $12 \mathrm{~km}$ from Kankavli, 13 Apr. 1971, B. G. Kulkarni 121196 (BSI); Amboli, Chaukul Road, 20 Apr. 1971, B. G. Kulkarni, 128829 (BSI); ibid, 29 Feb. 2016, M. D. Nandikar, 1451 (NGCPR); Choukul, Amboli, 18 Apr. 2017, D. C. Jadhav 1464 (NGCPR); Sawantwadi-Talkat road, 09 Apr. 2019, M. D. Nandikar 2444 (NGCPR). Odisha, s. loc., 1835, Roxburgh s. n. (K); Near Khurda, 5 Apr. 1917, s. coll. (K); Kuldhia, Tari, s. d., J.S. Gamble 9314 (K); Sambalpur, Kuldhia forest 29 Dec. 1943, H. F. Mooney 2379 (K); Kendrapara, Hukitola, 5 Feb. 1961, G. Panigrahi 23710 (CAL); ibid, 1 Nov. 1972, L. K. Banerjee 9425 (CAL); Mahanadi delta, Hetamunda, 16 June 1972, L. K. Banerjee 8976 (CAL); Kendrapara, Bhitarkanika, 18 Feb. 1973, L. K. Banerjee 10139, (CAL); ibid L. K. Banerjee 10124 (CAL); ibid, 05 Feb. 1961, G. Panigrahi 23710 (ASSAM, CAL); Shores of Chilkha Lake, 30 Dec. 1915, Haines 3899 (CAL). Tamil Nadu, s. loc., 1867, R. Wight 348 (K); s. loc, 1866, R. Wight 468 (K); Ponparappu, 29 Apr. 1896, s. coll., s. n. (K); Vilupuram, Marakkananam, 23 Feb. 1972, F. Blasco 2243 (K); Pudukottai, Kulathur, Narthamalai, 22 Mar. 1980, K. M. Matthew 27264 (K); Nagapattinam, Kodikerai, 5 Feb 1995, $H$. S. Suresh \& Rajan. J. 2719 (FRLH); Viluppuram, Vanur taluka, Marakkanam, 31 Jan. 1996, W. F. Gastmans 2941 (FRLH); Salem, Attur, Periakalrayans Nagalur Forests, 10 Mar. 1980, K. M. Matthew 26949; 26933 (RHT); ibid, 05 July 1979, K. M. Matthew 23568 (RHT); Salem, Attur, Periankalrayans, via Nagalur Reserve Forest to Kumakkal bridle path, 21 Sept. 1978, N. Venugopal \& C. Monoharan 17585 (RHT); Tiruchirappalli, Thuraiyur, Pachaimalais, top Sengattupatty Vengamudi path, 20 Mar. 1978, K. M. Matthew 12507 (RHT; CAL); ibid, 19 Mar. 1978, K. M. Matthew 12493 (CAL); ibid, 17 May 1976, K. M. Matthew 12360 (CAL); South Arcot, Chidambaram, Killai, opposite to Karithurai, 07 Oct. 1976, K. M. Matthew \& V. A. A. Raj 4326 (RHT); Kanchipuram, Tambaram, MCC Campus, 12 Mar. 1999, K. M. Matthew \& K. T. Augustine 60626 (RHT); ibid, 05 Jan. 2001, C. Livingstone 72082 (RHT); Dharmapuri District, Harur, Kottapatti, 16 May 1978, K. M. Matthew 14125 (CAL). Pondicherry, Auroville, Marakkanam, 27 May 1999, K. 
M. Matthew, K. T. Augustine \& W. F. Gastmans 60778 (RHT). West Bengal, Sundarban, 26 Jan. 1894, R. L. Heinig 22 (CAL); Midanpur, Tapoban, 07 May 1976, Sudarran Maiji 6498 (CAL); Sagar Island, 01 May 1967, A. K. Muk s. loc., s. d., V. Ball 177 (K).

\section{Notes}

The scandent habit of $S$. chinensis can often be seen along the riverbanks and the erect shrubby habit is abundant on the plateaus in Maharashtra, Karnataka, Kerala, and Tamil Nadu. It can be recognised by the few-flowered axillary and ramiflorous fascicles, smaller globose, 1-seeded fruit with thin exocarp and hyaline, pulpy endocarp.

Collections from the Andaman and Nicobar Islands are more likely to be of S. platyphylla, as the latter differs from $S$. chinensis in its broader leaves and 2-seeded berries. Salacia chinensis is similar to $S$. megacarpa in having obovate to oblanceolate petals, but the latter is clearly distinguished by its branched cymes and ellipsoid to prolate, ridged, dry fruits.

4. Salacia fruticosa Wall. ex M.A. Lawson in Fl. Brit. India 1(3): 628. 1875. (Figure 11)

Type: India, Karnataka, Wallich Cat. No. 4223, Herb Heyne s.n. (K! barcode K001038432) lectotype here designated; isolectotype CAL! barcode CAL87503).

(=) Christmannia courondi Dennst. ex Kostel. Allg. Med.-Pharm. Fl. 5: 2005. 1836. nom. illeg. (Nicolson and Suresh 1986).

Iconotype: 'Courondi' Rheede Hort. Malab. 4: t. 50. 1683.

\section{Description}

Scandent shrubs or lianas up to $2(-6) \mathrm{m}$ high; stems terete, branched, branchlets looped, glabrous. Leaves opposite to sub-opposite, exstipulate; petioles 5-6 mm; lamina ovate-broadly elliptic, $4-10(-12) \times 2-5 \mathrm{~cm}$, apex acute-acuminate, base obtuse-cuneate, margin usually crenate, rarely entire, midrib distinct, lateral nerves 5 or 6 pairs. Inflorescences axillary and ramiflorous, pedunculate, peduncle 5-10 mm long, 10-14-flowered cymes; buds globose; pedicel 1-1.2 cm long, glabrous; bracts sepaloid; sepals fused, lobes oblong, 0.3-0.4 $\times 0.4 \mathrm{~mm}$, undulate, crisped, white, faintly ciliate; petals orbicular, $1.5 \times 1.5 \mathrm{~mm}$, ochreous, margin faintly hyaline; disk flat to annular, fleshy, orange or ochreous; stamens 3 , inserted on disk, filaments linear to clavate, white; anthers bilobed, ovoid, yellow, thecae confluent longitudinally; ovary globose, 3 -celled, style short, stigma simple. Fruits

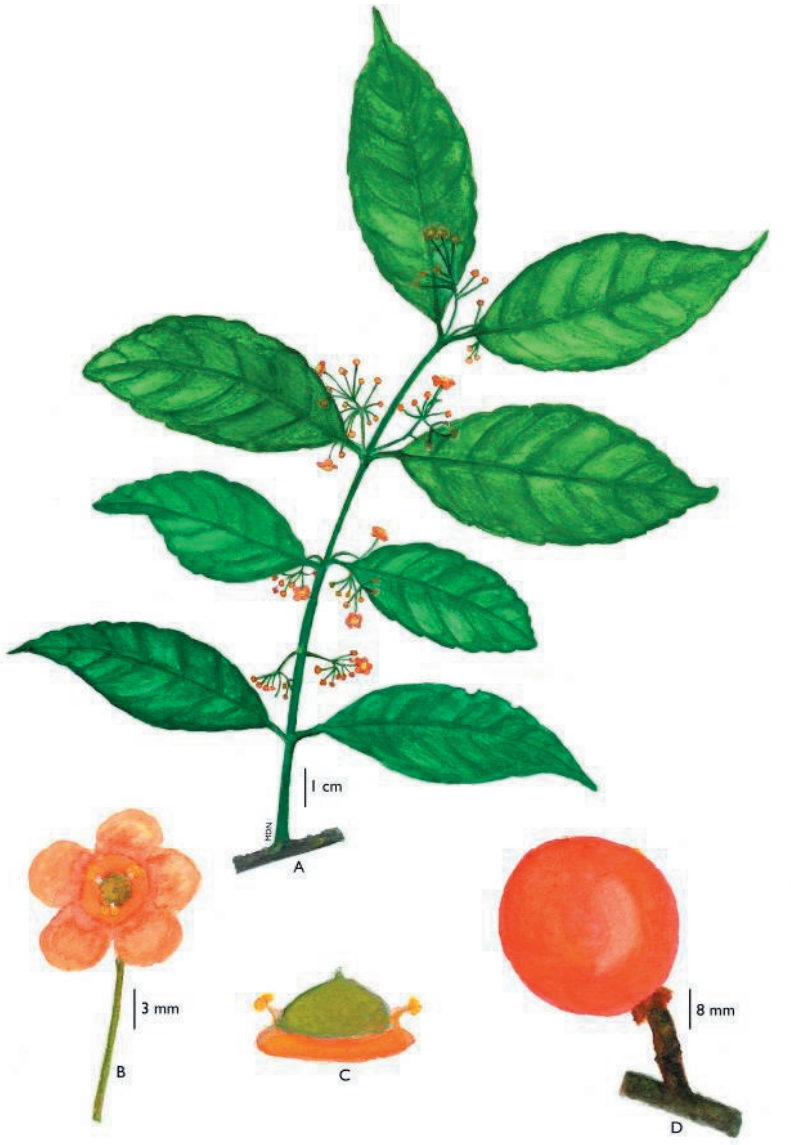

Figure 11. Salacia fruticosa (A) Flowering twig. (B) Flower. (C) Disc. (D) Fruit

baccate, shortly stipitate, globose, $2.5-3.5 \times 2-3 \mathrm{~cm}$, exocarp smooth, orange when ripening, endocarp mucilaginous, 2- or 3-seeded; seeds angular, ovoid, or ellipsoid, $1.5-2 \times 1 \mathrm{~cm}$, peach coloured.

\section{Phenology}

Flowers in January, fruits may remain till end of August, rarely till November.

\section{Distribution and habitat}

Endemic to the Western Ghats of Karnataka, Kerala, and Tamil Nadu (Figure 18). It prefers semi-evergreen to evergreen fringing forest, also known from coastal scrubland.

\section{Vernacular name}

"korandi" (Tamil, Malayalam)

\section{IUCN status}

S. fruticosa is recorded from several localities in the 
states of southern Western Ghats with strong and viable populations. Currently, no threats have been observed or recorded and no plausible future threats are recognised. Therefore, it is assessed as Least Concern (LC).

\section{Specimens examined}

INDIA. Karnataka, Coorg, Mar. 1817, Herb. Rottler ex Heyne s.n. (K [K000687001]); Mangalore, Jan. 1847, Hohenacker s. n. (K); Udupi, Brahmavara, 2 May 1980, C.J. Saldanha 11366 (CAL); Dakshin Kannada, Vittala, 24 Mar. 2016, D. C. Jadhav 1452 (NGCPR). Kerala, Quilon (Kollam), 1835, R. Wight 467 (K); s. loc., 5 Apr. 1895, T. F. Bourdillon 570 (K); Wayanad, Thambarcheri Ghats, 21 Jan. 1903, C. A. Barber 5679 (K); Kasargod, 22 Feb. 1913, C. A. Barber 8810 (K); Thenmala, Ariyankavu, 9 Mar. 1980, C. E. Ridsdale 540 (K); Kannur District, Kannoth R.F., 16 Feb. 1978, s. coll. 54009 (CAL); Kannur, Aralam, 26 Feb. 1979, V. S. Ramachandran 61928 (CAL); Kasargod, Panathur, 29 June 1980, R. Ansari 67913 (CAL); Kasaragod, Sreekantapuram, 15 May 1982, V. J. Nair 73097 (CAL); Pathanamthitta, Aryattukavu, Nilakkal, 26 Feb. 1988, Anil Kumar 496 (CAL); Kasargod, Mulleria, 8 May 1982, U. J. Nair 7105 (CALI); Idukki, Painavu, 27 Apr. 1984, C. N. Mohanan 81637 (CALI); Palakkad, Panthenthode border, 8 May 1984, T. Sabu 11444 (CALI); Malappuram, Calicut University Campus, 12 Feb. 1989, Q. P. Elsie 43120 (CALI); Pathanamthitta, Moozhiyar forest, 8 June 1990, R. Chandrasekaran 94010 (CMPR); Idukki, Elappara, 22 Sep. 2005, P. S. Udayan 3629 (CMPR); Chandanathode, 20 Apr. 2010, P. S. Udayan 6716 (CMPR); Kannur, Udayagiri 5 Jan. 2009, P. S. Udayan 5377 (CMPR); Kollam, Kulathupuzha 7 Feb. 2008 P. S. Udayan 4503 (CMPR); Kozhikkode, Koorachurdu, 19 May 2003, P. S. Udayan 1671 (CMPR); Kozhikkode, Jeerakappra 22 July 1994 A. Nazarudeen 22753 (TBGRI); Kottayam, Peringulam, 11 Apr. 2009, A. J. Robi 5885 (CMPR); Ammayambalam pacha, 2 July 1992, A. Nazarudeen 14345 (TBGRI); Kollam, Arienkavu, 24 Sep. 1993, A. Nazarudeen 18060 (TBGRI); Kasargoad, Edaneer kavu, 12 Mar. 1993 A. Nazarudeen 16418 (TBGRI); Malappuram, Kottakkal, CMPR Herbal Garden, 26 Mar. 2016, D. C. Jadhav, 1453 (NGCPR); Pathanamthitta, Attathode, 20 Mar. 1994, A. Nazarudeen 20302 (TBGRI); Kottavasal, 28 June 1994, A. Nazarudeen 21796 (TBGRI); Pangappara, 10 May 1994 A. Nazarudeen 18779 (TBGRI); Kollam, Thenmala, 25 Nov. 2004, Geethakumary 55375 (TBGRI); Dharpakulam, 29 May 2012, Deepthy \& Usha 76215 (TBGRI); Thiruvananthapuram, Pallode, 15 Feb. 1996, P. Chiranjit 126 (K); Trissur, Peechi, 16 Mar. 1996, P. Chiranjit 130 (K); Thiruvananthapuram, Palode, JNTBGRI garden, 21 Feb. 2014, S. M. Shareef 79239 (TBGRI); Idukki, Laksh- mi Estate, 30 Dec. 2014, Deepthy \& Jaya 76210 (TBGRI); Thiruvananthapuram, JNTBGRI Garden, Palode, 16 Feb. 2017, D.C. Jadhav 1460 (NGCPR); Kozhikode, Kakkayam Ghat, 6 Mar. 2018, D. C. Jadhav \& M. D. Nandikar 1484 (NGCPR); KFRI Garden, Trissur, Aug. 2016, K.C. Kishor s. n. (NGCPR00509; NGCPR00510). Tamil Nadu, Annamalai Hills, 1885, R.H. Beddome s. n. (K); Kanyakumari, Lower Kodayar, Vallachithode, 28 Mar. 1981, A. N. Henry 70665 (CAL); Karur, Devarmalai, 13 Aug. 1988, R. Chandrasekaran 89205 (CAL); Kanyakumari, Kulasekaram, Pechiparai, 17 Mar. 1994, S. P. Subramani 560 (FRLH).

\section{Notes}

Salacia fruticosa can be recognised in the field by its scandent habit, ovate-elliptic, crenate leaves, acuteacuminate apex, pedunculate dichotomously branched cymes, orbicular petals, and smooth, orange fruits. It was first described as Tonsella fruticosa in Wallich Catalogue (no. 4223), based on Heyne's collection and later validated by Lawson citing additional collections by Rottler, Hoenacker, and Kurz. The collection by Heyne here designated as lectotype was cited in Wallich Cat. 4223 and agrees with the protologue.

Rheede's (1683) 'Courondi' was previously attributed to Salacia chinensis (Ding Hou 1964, Nicolson \& Suresh 1986). However, the branched cymes and orbicular petals illustrated by Rheede's artists agree more with $S$. fruticosa (a much more common species in the Malabar region) than S. chinensis.

5. Salacia gambleana Whiting \& Kaul in Bull. Misc. Inform. Kew 1940(7): 300. 1941. (Figure 12)

(=) Salacia talbotii Gamble in Bull. Misc. Inform. Kew 1916(5): 133. 1916 nom. illeg., non Baker Cat. Pl. Oban 19: 1913.

Type: India, Karnataka, North Canara, Anshi Ghat, 2 June 1885, W. A. Talbot 1217 (lectotype K! barcode K000669981), designated by Jadhav et al. (2016).

\section{Description}

Scandent shrubs or spreading lianas up to $4(-7)$ $\mathrm{m}$ high; branchlets looped, lenticellate. Leaves simple, opposite to subopposite, exstipulate; petiole $1-1.5 \mathrm{~cm}$ long; lamina usually oblanceolate, rarely elliptic-oblong, 6-12 × 3-4 cm, apex abruptly acute, base attenuate, margin obscurely undulate, recurved, glabrous, lateral nerves 6-8-paired. Inflorescences axillary, ramiflorous, few to many-flowered, tuberculate fascicles; buds glo- 


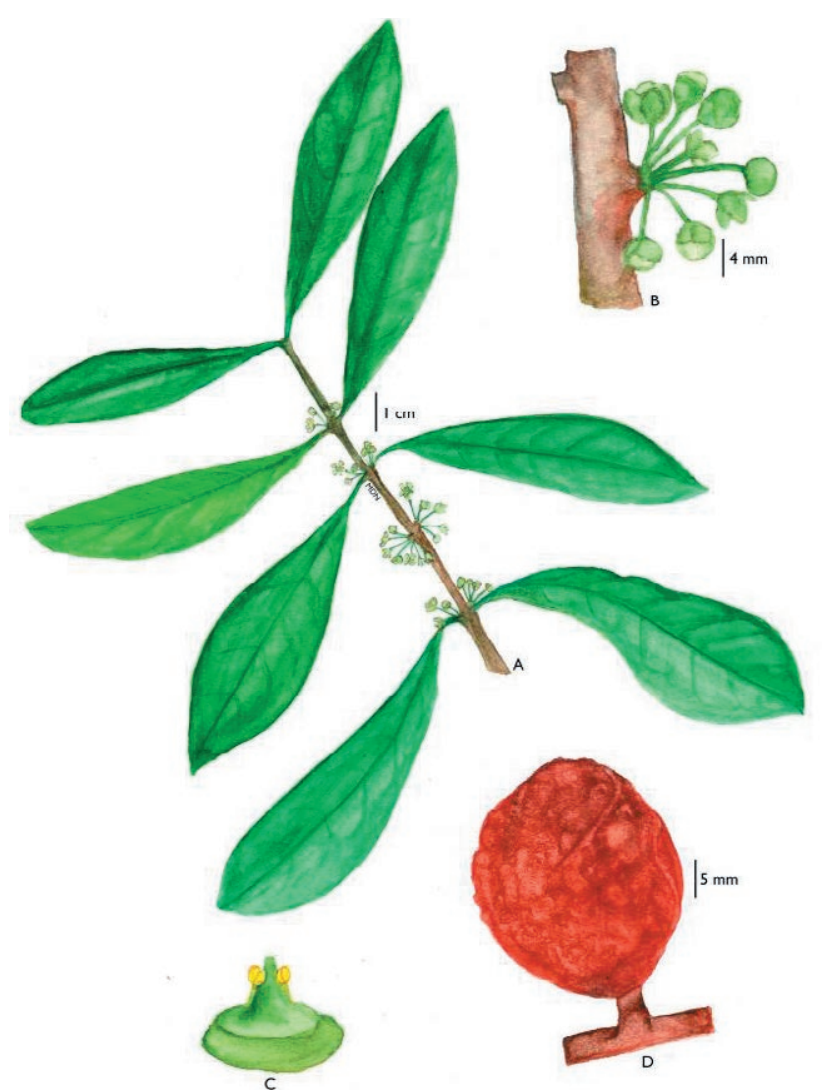

Figure 12. Salacia gambleana (A) Flowering twig. (B) Disc. (C) Inflorescence. (D) Fruit.

bose; pedicels glabrous, c. $8 \mathrm{~mm}$ long; sepals ovate, c. $1.5 \mathrm{~mm}$ long, margin laciniate to fimbriate, pale green; petals elliptic to ovate, c. $4 \mathrm{~mm}$ long, obtuse, coriaceous, pale yellow to green, margin hyaline; disk conical or annular, greenish yellow; stamens 3 , inserted on inner margin of the disc, anthers bilobed, globose, yellow, thecae confluent transversely; ovary embedded in disc, 3-celled, style short, conical, exserted; stigma inconspicuous. Fruits baccate, globose to ellipsoid, $2-3 \mathrm{~cm}$ across, exocarp smooth to verrucose, often tuberculate, orange when ripen; endocarp mucilaginous, 2-5-seeded; seeds dorsiventrally compressed or discoid, oblong or ellipsoid, or oblate c. $10 \times 7 \mathrm{~mm}$, peach coloured.

\section{Phenology}

Flowers between January to April, fruits may remain till July.

\section{Distribution and habitat}

Endemic to the Western Ghats of Karnataka and Maharashtra (Figure 18). Evergreen forest, fringing forest, at $550 \mathrm{~m}$ a.s.l.

\section{IUCN status}

S. gambleana is occasionally found in the forests of north Karnataka and western Maharashtra. The species occurs at considerable number of localities and no threat is known at present. Hence, it is assessed here as List Concern (LC). However, more detailed study on population size and threats are needed to have precise IUCN status. Although the species was assessed as Vulnerable (VU) by Kamat et al. (2020), no data on population size and threat were provided by them to support their assessment.

\section{Specimens examined}

INDIA. Karnataka, North Canara, Anshi Ghat, 25 Jan. 1886, W.A. Talbot 1361 (K, CAL); Agumbe, 15 May 1960, R.S. Raghavan 62515 (CAL); Barakana, Agumbe, 13 June 1961, R.S. Raghavan 74211 (CAL, BSI); Minhole, Agumbe, Shimoga, 19 Feb. 1963, R.S. Raghavan 86353 (BSI); Agumbe, 28 Mar. 2016, D.C. Jadhav 1456 (NGCPR); Basanbail village, 21 Apr. 2017, D.C. Jadhav 1471 (NGCPR); ibid, D.C. Jadhav 1472 (NGCPR); Shimoga, Sharavati River Valley viewpoint, 21 Apr. 2017, D. C. Jadhav 1476 (NGCPR); Anshi Ghat, 20 Apr. 2017, D.C. Jadhav 1468 (NGCPR). Maharashtra, Kolhapur, Dajipur, 3 Jul. 2016, M.D. Nandikar 1457 (NGCPR).

Note

Salacia gambleana is mostly confused with S. macrosperma due to the similar habit and floral characters. However, the latter can be differentiated by its ellipticoblong leaves, and 6-12-paired lateral nerves. I have examined several collections (from Maharashtra, Karnataka, and Kerala) at CAL, BSI, and $\mathrm{MH}$ that were wrongly identified as S. macrosperma and S. beddomei. The oblanceolate leaves and 6-8-paired lateral nerves are amongst the more reliable characters to separate $S$. gambleana from allied species. Most collections from Agumbe, Karnataka clearly belong to S. gambleana.

6. Salacia macrosperma Wight, Spicil. Neilgh. i. 28. t. 32. October 1845; Icon. Pl. Ind. Orient. 3(3): 5, t. 962. November 1845. (Figure 13)

Type: India, Kerala, Sispara peak, s. d., Wight s. n. (K! barcode K000669996), lectotype here designated.

(=) Salacia beddomei Gamble in Bull. Misc. Inform. Kew 1916(5): 133. 1916 syn. nov.

Type: India, Tamil Nadu, Coimbatore District, Annamalai hills, 1866, R. A. Beddome s.n. (lectotype K! 


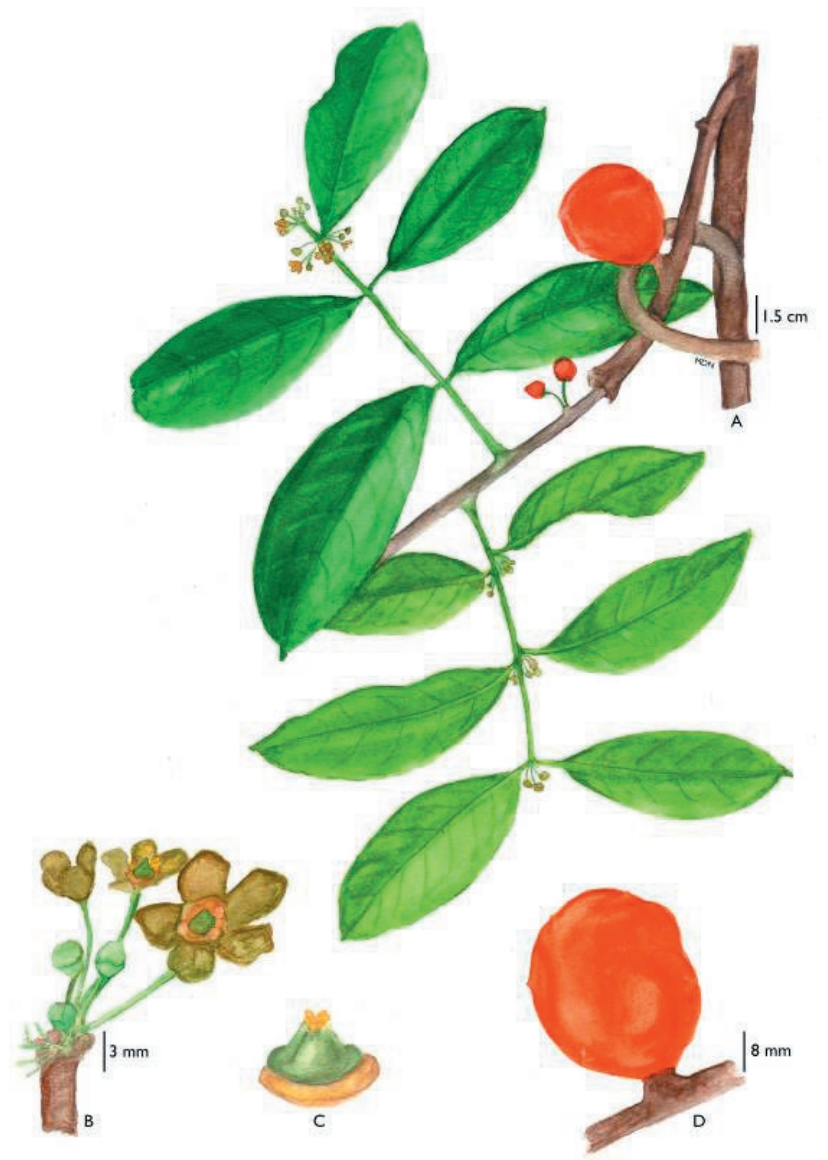

Figure 13. Salacia macrosperma (A) Flowering twig. (B) Disc. (C) Inflorescence. (D) Fruit.

barcode K000669921; isolectotype MH! barcode MH00001914), designated by Jadhav et al. (2016).

(=) Salacia vellaniana Udayan, Yohannan \& Pradeep in Candollea 68(1): 148. 2013 syn. nov.

Type: India, Kerala, Thrissur District, Vellanipacha 26 Feb. 2011, Udayan S005 (holotype CALI; isotypes MH, $\mathrm{SKC}, \mathrm{SNCH}$ ).

\section{Description}

Scandent shrubs or straggling lianas up to 2(-9) $\mathrm{m}$ high; branched, branchlets thick, grey to brown glabrous to lenticellate, distal branches spreading. Leaves opposite to sub-opposite, exstipulate, petiole $0.5-1 \mathrm{~cm}$ long; lamina elliptic-oblong or obovate-elliptic, $8-15 \times$ 3-7 cm, apex acute to acuminate, base narrowly cuneate, rounded-obtuse, margin entire, rarely serrate to crenate, midrib distinct, venation anastomosing, lateral nerves 6-12 pairs. Inflorescences axillary, ramiflorous, $4(-7)$ to many-flowered, tuberculate fascicles; buds glo- bose, flowers pedicellate, pedicel 5-9 $\mathrm{mm}$ long, glabrous; bract sepaloid; sepals fused at base, lobes ovate-oblong, 0.8-0.6 mm long, laciniate to fringed; petals ovate to elliptic, $1-1.9 \times 1.2-1.3 \mathrm{~mm}$, pale green-yellow, margin hyaline; disk flat, annular, pale-yellow; stamens 3 , inserted on the disk, filaments short, broad at the base, anthers bi-lobed, globose, yellow, thecae transversely confluent; ovary 3-celled; style short, conical, c. $0.4 \mathrm{~mm}$ long; stigma simple. Fruits baccate, globose to ellipsoid, $2.5-4 \times 2-3$, exocarp orange when ripen, smooth to faintly tuberculate, or smooth, endocarp mucilaginous, 3-6-seeded; seeds ovoid, ellipsoid, often discoid, c. $2.5 \times$ $1.5 \mathrm{~cm}$, ochraceous.

\section{Phenology}

Flowers between February to April and fruits may remain till August.

\section{Distribution and habitat}

Endemic to the Western Ghats of Goa, Maharashtra, Karnataka, Kerala, and Tamil Nadu (Figure 19). Straggling over small trees in semi evergreen fringed forest, along roads, often grows as large shrubs with distal scandent branches along the edges of plateaus.

\section{IUCN status}

S. macrosperma is widely distributed in the Western Ghats, and a good and stable population trend was observed during the field surveys. Also, no major threats or future obstacles were detected. Hence, it is assessed here as Least Concern (LC).

\section{Specimens examined}

INDIA. Goa, Molem, Anomd Ghat (Karnataka Border), 19 Apr. 2017, D. C. Jadhav 1466 (NGCPR); ibid, Anmod Ghat, D. C. Jadhav 1467 (NGCPR). Maharashtra, Sindhudurg, Amboli, Mahadeogad, 4 Sep. 1968, B. G. Kulkarni 108678 (BSI); Sindhudurg, Amboli, 22 Apr. 1971, B. G. Kulkarni 128874 (BSI). Karnataka, Belgaum, Yadur village, 11 Feb. 1963, R. Raghavan 86009 (BSI); Belgaum, Londa, Anmod Road, 22 May 1979, C. J. Saldanha 7817 (CAL); Chorla Ghat, 31 June 2017, M. D. Nandikar 1533 (NGCPR); Hassan, Between Devalkere \& Devarunde, 19 Feb. 1972, Ramamoorthy \& K. N. Gandhi 2674 (K); ibid, 14 Apr. 1969, C. J. Saldanha 13338 (K); Agumbe, Nishanigudda Road, 19 May 1960, R. Raghavan 62737 (A); Agumbe, Ghatibagh, 15 May 1950, R. Raghavan 80614 (BSI); Shimoga, Agumbe, Cattle shed, 17 May 1960, R. Raghavan 62599 (CAL); Nallur, 7 Feb. 1961, R. Raghavan 69442 (BSI); Kalmane, 19 May 1962, R. Raghavan 80765 (BSI); ibid, 13 Feb.1963, 
R. Raghavan 86225 (BSI); Shimoga, Agumbe Ghat, 28 Mar. 2016, D. C. Jadhav 1456 (NGCPR); Yana Ghats, on the way to Vaddi village, 21 Apr. 2017, D.C. Jadhav 1469 (NGCPR); Malemande, 21 Apr. 2017, D. C. Jadhav 1475 (NGCPR); Thalacauvery, 04 Mar. 2018, M. D. Nandikar \& D. C. Jadhav 1482 (NGCPR); Devimane Ghats, 22 Apr. 2017, D. C. Jadhav 1478 (NGCPR); Karikanamma, 22 Apr. 2017, D. C. Jadhav 1477 (NGCPR); Basanbail, 02 March 2018, D. C. Jadhav 1480 (NGCPR); Shimoga, Sharavathi River, Valley viewpoint, 21 Apr. 2017, D. C. Jadhav 1474 (NGCPR); Chorla Ghat, 03 June 2017, M. D. Nandikar 1532 (NGCPR); Yana Ghat, on the way to Vadi Village, 21 Apr. 2017, D. C. Jadhav 1470 (NGCPR), Kerala, Kakkayam Dam Site, 06 Mar. 2018, D. C. Jadhav \& M. D. Nandikar 1486 (NGCPR); Malappuram, Calicut, 20 Feb. 2010, M. S Devipriya \& Regy Yohannan CNCHS3 (MH). ibid, 25 Jan. 2006, P. S. Udayan 3978 (CMPR); Malappurum, Kottakkal, Herb Garden, 26 Mar. 2016, D. C. Jadhav 1454 (NGCPR); Malappurum, Kottakkal, Herb Garden, 15 Jan. 2007, P. S. Udayan 5026 (CMPR); ibid, 23 Jan. 2008, P. S. Udayan 4380 (CMPR); Kozhikkode, Vellarimala, 1 Feb. 2008, P. S. Udayan 4499 (CMPR); Kannur, Aralam, 23 Feb. 2008, P. S. Udayan 4749 (CMPR); Malappuram, Anoli 4 Mar. 2009, A. J. Robi. 5714 (CMPR); Kozhikode, Kakkayam, 5 June 2008, P. S Udayan 5102 (CMPR); Palghat, Panthanthode to Dam site via old road, 3 Apr. 1983, N. C. Nair 77279 (K); ibid 3 Apr. 1983 N. C. Nair 77282 (K); ibid, 3 Apr. 1983, N. C. Nair 77279 (MH); Palghat, Silent valley, 8 Feb. 1995, N. Sasidharan 10687B (FRLH); Palode, 10 Apr. 1992, A. Nazarudeen 14132 (TBGRI); Palode, 24 Feb. 1993, A. Nazarudeen 16156 (TBGRI); Thiruvananthapuram, near damp site TBGRI, 30 Jan. 1996, Jothish P. S. 27634 (TBGRI); Thiruvananthapuram, Vitalapacha, 18 Feb. 1992, A. Nazarudeen 13846 (TBGRI); Palghat, Aruvappra 19 Nov. 1993, A. Nazarudeen, 18722 (TBGRI); Kozhikkode, Jeerakappra, 22 July 1994, A. Nazarudeen 22752 (TBGRI); Palakkad, Poochapara, 6 Mar. 2009, A. J. Robi 5685 (CMPR); Palakkad, Mukkali forest, 7 Mar. 1975, E. Vajravelu 49782 (CAL, MH); ibid, 18 July 1969, E. Vajravelu 32184 (MH); ibid, 7 Mar.. 1975, E. Vajravelu 46233 (MH); Panthenthode, 10 Apr. 1978, N. C. Nair 56730 (MH); Kollam, Chandanathope, 30 Apr. 1979, V. S. Ramachandran 61655 (MH); Mukkali forest, E. Vajravelu, 33319 (MH); Kollam District, s.l. 18 Feb. 2010, Devipriya M.S \& Regy Yohannan, s.n. (MH); Thrissur District, Vellanimala, 18 Apr. 1984, N. Sasidharan 3079 (KFRI); ibid, 10 May 1985, N. Sasidharan 3484 (KFRI); ibid, 13 Mar. 1987, N. Sasidharan 3940 (KFRI); ibid, 11 Jan. 2013, N. Sasidharan 27903 (KFRI); ibid, 23 Feb. 2005, P.S. Udayan et al. 03371 (CMPR); ibid, 20 June 2009, P.S. Udayan et al. 06121 (CMPR).
Note

In addition to Salacia gambleana (see the note under S. gambleana), S. macrosperma also resembles S. salacioides in having entire leaves, axillary fascicles and ovoid fruits. However, the latter differs by its drooping branches, stipulate, broadly lanceolate leaves and orbicular petals. Salacia macrosperma is an extremely variable species, particularly in its leaf size, number of lateral nerves, and smooth to faintly tuberculate fruits. Some variants earlier described as separate species have been compared based on their protologues, and live collections of S. macrosperma from Kerala. The analysis reveals the description of $S$. macrosperma to be a perfect match for S. beddomei and S. vellaniana. Salacia beddomei Gamble (1916) was described based on Beddome's collection from Anamalai Hills, Tamil Nadu. The species was differentiated from $S$. macrosperma by having larger leaves with prominent lateral nerves and reddish brown, fimbriate sepal margins. I had an opportunity to observe the original material of S. beddomei at K (K000669921) and MH (MH00001914), and to compare the type of S. macrosperma. After studying the protologue, illustration made by Gamble on the type sheet revealed that the characters used to describe $S$. beddomei matches those of $S$. macrosperma, hence it is here relegated to synonymy. Unfortunately, Gamble (1916) had not seen the fruits for S. beddomei and this might have led him to misinterpret the species. Salacia vellaniana from Vellanimala, Thrissur, Kerala (Udayan et al. 2013) is based on characters such as smaller leaves, short petiole, few-flowered cymes, colour of the petals and sepals, and warty fruits. All these characters are within the variation range for $S$. macrosperma.

7. Salacia malabarica Gamble in Bull. Misc. Inform. Kew 1916(5): 133. 1916. (Figure 14)

Type: India, Kerala, Kollam District, Travancore Hills, Nov. 1887 T. F. Bourdillon 18 (MH! barcode MH00001913), lectotype designated by Jadhav et al. (2016).

\section{Description}

Scandent shrubs or lianas up to $2(-5) \mathrm{m}$ high; branchlets purplish, terete, glabrous or lenticellate, grey to brown. Leaves opposite, exstipulate, petiole $1-1.2 \mathrm{~cm}$ long, lamina elliptic-oblong or obovate, $8-16 \times 3.5-7$ $\mathrm{cm}$, apex obtuse-acute, base shortly attenuate or obtuse, margin distinctly serrate, lateral nerves 7-10 pairs. Inflorescences axillary, ramiflorous, 2-many-flowered, tuberculate fascicles; pedicel $1.5-2(-2.5) \mathrm{cm}$ long, glabrous; sepals fused at base, lobes ovate, $3 \mathrm{~mm}$ long, entire to sparsely ciliate, pale green; petals elliptic-oblong, 5-7 


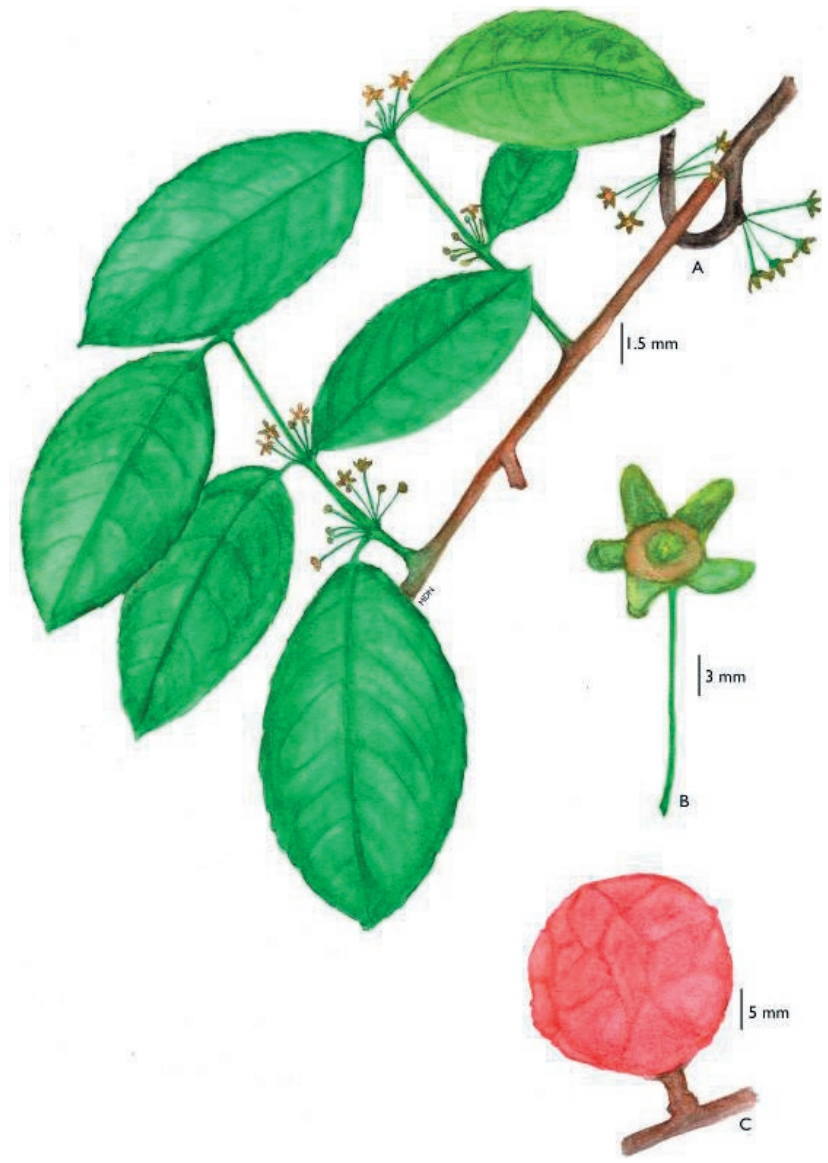

Figure 14. Salacia malabarica (A) Flowering twig. (B) Disc. (C) Fruit.

$\times 2-3 \mathrm{~mm}$, green, margin greenish yellow; disc yellowochreous, conical, stamens inserted between lobes of disc, filaments usually recurved, rarely porrect, short, flattened, anthers globose, yellow, thecae transversely confluent; ovary embedded in the disc, 3-celled, style conical, stigma simple. Fruits baccate, globose, 1.5-2.5 $\mathrm{cm}$ in diameter, exocarp verrucose, orange at maturity, endocarp mucilaginous, 3-6-seeded; seeds ovoid, ochreous to brown.

\section{Phenology}

Flowers from November, peak in February and fruits may remain till June.

\section{Distribution and habitat}

Endemic to the Western Ghats of Karnataka and Kerala (Figure 19). It grows in evergreen and moist deciduous forest, coastline scrubland and fringing forest. Its occurrence in southeast Asia as recorded by Kamat et al. (2020) is wrong.

\section{IUCN status}

S. malabarica is sporadically distributed in coastal districts of Kerala and south Karnataka. The southernmost distribution is Kottoor Reserve Forests, whereas the northernmost distribution is known from Dakshin Kannada District. As much of the known populations falls within the Biosphere Reserves, hitherto no threat has been observed and the species is seemingly secured and protected. Hence, it is assessed here as Least Concern (LC). The IUCN status as Endangered (EN) by Kamat et al. (2020) is flawed and contradicts their remarks on the species' distribution.

\section{Specimens examined}

INDIA. Kerala, Palghat, Silent Valley, 25 Jun. 1976, C. E. Ridsdale 285 (K); Kollam, Travancore Hills, Nov. 1887, T. F. Bourdillon 18 (MH); ibid, 25 Nov. 1893 T. F. Bourdillon s.n. (MH [MH00001912], K [K000669995]); Kollam, Kattlapara to Choodal, Shendurney Wildlife Sanctuary, 7 Feb. 2008, P. S. Udayan 4512 (CMPR); Wayanad, 13 Apr. 2009, M. S. Devipriya \& Regy Yohannan 171691 (MH); Wayanad, Kuruva Island, 5 Apr. 2009, A. J. Robi 5835 (CMPR); Thiruvananthapuram, JNTBGRI, 17 Jan. 2014, S. M. Shareef 79215 (TBGRI); ibid, 13 Feb. 2014, S. M. Shareef 79227 (TBGRI); Agasthyavanam, Kottur Reserve Forest, 3 Apr. 1973, J. Joseph 44010 (MH); JNTBGRI, Palode, 16 Feb. 2017, D. C. Jadhav 1461 (NGCPR). Karnataka, South Canara, s.d., Beddome s. $n$. (MH); South Canara, Subramanya, 25 Feb. 1997, S. R. Ramesh 9925 (FRLH).

Note

S. malabarica can be recognised by its ellipticoblong or obovate, distinctly serrate leaves, $2-12$, pedicellate (1.5-2 cm long) flowers on axillary, ramiflorous, tuberculate, fascicles. It shares similarities with $S$. gambleana and $S$. macrosperma but differs in having broadly elliptic to oblong, serrate leaves and long pedicellate flowers. Udayan 04512 (CMPR) from Shendurney Wildlife Sanctuary, has entire to faintly serrate leaves, and c. $2.5 \mathrm{~cm}$ long fruiting pedicel, but it appears to agree with $S$. malabarica in most of the other diagnostic characters of the species.

8. Salacia megacarpa N.V.Page \& Nandikar in Nordic J. Bot. 38(4)-e02647: 2. 2020. (Figure 15)

Type: India, Karnataka, Abailu Antipoaching Camp, Bramhagiri Wildlife Sanctuary, Virajpeth Taluk, Kodagu District, 850 m. a.s.l., 23 Jan. 2016, N.V. Page 22201 (holotype: $\mathrm{MH}$, isotypes: JCB, WII, NGCPR). 


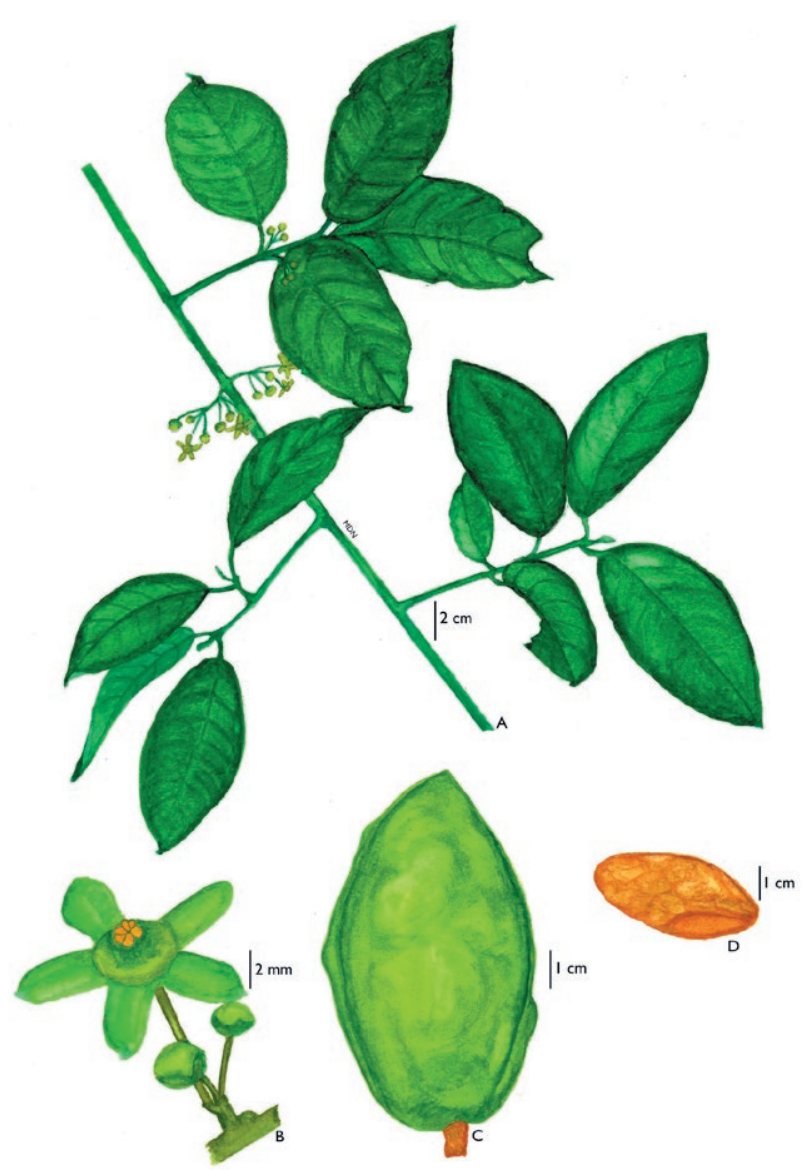

Figure 15. Salacia megacarpa (A) Flowering twig. (B) Inflorescence. (C) Fruit. (D) Seed

\section{Description}

Scandent shrubs or spreading lianas up to $2(-4) \mathrm{m}$ high; branches slender, hooked, glabrous, sparsely lenticellate. Leaves opposite, stipules inconspicuous or absent; petiole $1-1.5 \mathrm{~cm}$ long, lamina broadly ellipticovate, oblong, 9-17 $\times 3.5-8 \mathrm{~cm}$, apex rounded to obtuse or acute, base cuneate or rounded, margin entire or faintly crenate, coriaceous, surface glabrous, adaxial dark green and abaxial light green; midrib and lateral nerves 6-10 in pairs and prominent with brown after drying. Inflorescences axillary and ramiflorous, 3-6 flowered cymes, peduncle c. $5 \mathrm{~mm}$ long. Flower buds spherical or globose, citrine, with calyptriform sepals; flowers c. $10 \mathrm{~mm}$ wide, dark greenish yellow, pedicel 3-5 mm long, sparsely puberulous, bracts minute, pedicel base with 1 or 2 inconspicuous rudimentary flowers with 2-4 petal whorls; sepals fused at base, lobes ovate, c. $1 \times 1 \mathrm{~mm}$, margin sparsely erose; petals elliptic-oblong, $3-4 \times 2 \mathrm{~mm}$, grey-green to yellowish green; apex rounded or acute, hyaline or erose, margin entire, hyaline, often revolute; disc pentagonous, pulvinate, c. 2 $\mathrm{mm}$ high, carnose, yellowish green; stamens 3, recurved, erect at anthesis, filaments short, c. $0.7 \mathrm{~mm}$ in length, with dilated bases; anthers bilobed, saffron or lemon, ovoid, thecae confluent obliquely; pistil pyramid shaped, cleft into 3, stamens emerging from each cleft of pistil; style c. $0.6 \mathrm{~mm}$ long; stigma simple; ovary embedded in disk, tri-locular. Fruits baccate, shortly stipitate, prolate to broadly ellipsoid, or ovoid, 7-10 $\times 4-6 \mathrm{~cm}$, distinctly 2- or 3-keeled, apex acute, greenish yellow, exocarp thin, endocarp dry, 5-8-seeded; seeds triangular to ovoid or ellipsoid, truncate (on hilum side), c. $3 \times 2 \mathrm{~cm}$, without mucilaginous pulp, ochreous, surface glabrous and with faint reticular meshes, hilum linear, sericeous.

\section{Phenology}

Flowers between January March, followed by fruiting till June.

\section{Distribution and habitat}

Endemic to the Western Ghats of Karnataka and Kerala (Figure 19). It grows in open and semi evergreen forest, fringing forest, along the perennial streams.

\section{ICUN status}

The conservation status is assessed as Least Concern (LC). At present the species is known from two localities in Karnataka and Kerala. No threats have been observed as both the locations are well protected and forms part of a Biosphere Reserve.

\section{Specimens examined}

INDIA. Kerala, Kozhikode, Malabar Wildlife Sanctuary, Kakkayam Dam, 6 Mar. 2018, D. C. Jadhav \& M. D. Nandikar 1485 (NGCPR, BSI). Karnataka, Kodagu, Virajpeth, Abailu Antipoaching Camp, Bramhagiri Wildlife Sanctuary, 16 May 2017, N.V. Page 22201B (WII, fruit: spirit collection).

\section{Note}

Lack of mucilaginous or fleshy seeds or endocarp in the fruits of Salacia megacarpa makes its distinct among its congeners. All the Salacia species in the study area have orange-ochreous, tawny fruits at the maturity, except the present species in which the fruits remain are green or greenish yellow at maturity. One of the largest fruits (c. 6 $\mathrm{cm}$ long) in the genus was described from the Malaysian S. maingayi M.A. Lawson (Ding Hou 1964). However, S. megacarpa fruits are claimed to be the largest in Salacia and is also distinct due to the presence of three keels running along the longitudinal axis of the fruit. 
Salacia megacarpa is similar to S. fruticosa with respect to its habit and inflorescence but the later differs in having crenate leaf margins, orbicular petals and 2- or 3-seeded, orange berries and mucilaginous pulpy seeds. Salacia oblonga also has axillary and ramiflorous, dichotomously branched cymes. The latter can, however, be easily differentiated from the species described here by having subsessile, urceolate flowers, globose and orange-red fruit. The IndoMalaysian S. korthalsiana also has similar branched cymes but can be distinguished based on single-seeded fruits. The Southeast Asian S. maingayi is the only other species which has comparable sized large fruits; however, it can be distinguished based on 1 or 2 axillary flowers.

9. Salacia oblonga Wall. ex Wight \& Arn., Prodr. Fl. Ind. Orient. 1: 106. 1834. (Figure 16)

Type: India, Wallich Cat. no. 4226, Herb Wight 351 (E! barcode E00174247), lectotype here designated.

(=) Salacia oppositifolia Rottler ex Wight \& Arn. Prodr. Fl. Ind. Orient. 1: 106. 1834 pro syn., nom. inval.

(=) Salacia oblonga. var. kakkayamana Udayan \& Raghu in Int. J. Pl. Anim. Environm. Sci. 4(3): 100. 2014. syn. nov.

Type: India, Kerala, Kozhikkode, Malabar Wildlife Sanctuary, Kakkayam, 12 Mar. 2012, P.S. Udayan, A.V. Raghu \& E.M. Muraleedharan 27802 (holotype CAL; isotypes MH, CALI, SKC, KFRI).

Salacia pomifera Wallich Numer. List no. 4227. 1831. nom. nud.

\section{Description}

Climbing or straggling shrubs or small trees up to $1(-3) \mathrm{m}$ high; young branchlets terete, rusty, abruptly yellow-corrugated. Leaves opposite or sub-opposite, petioles c. $1 \mathrm{~cm}$ long, lamina elliptic-oblong, rarely, oblanceolate, $7-12.5 \times 2.5-3.2 \mathrm{~cm}$, apex obtusely acuminate, base obtuse, margin entire, coriaceous, adaxial surface emerald, abaxial surface citrine, drying yellow, midrib distinct, venation anastomosing, lateral nerves 5-6 in pairs. Inflorescences axillary, ramiflorous branched, subsessile (peduncle c. $5 \mathrm{~mm}$ long), 5- or 6-flowered cymes; flowers urceolate; sepals fused at base, lobes ovate to orbicular, c. $2 \times 2 \mathrm{~mm}$, apex rounded, margin sparsely erose; petals 5 , free, oblong, c. $4 \times 3 \mathrm{~mm}$, yellowish green, apex rounded, margin entire, chartaceous; disk conical, pale green; stamens 3 ,

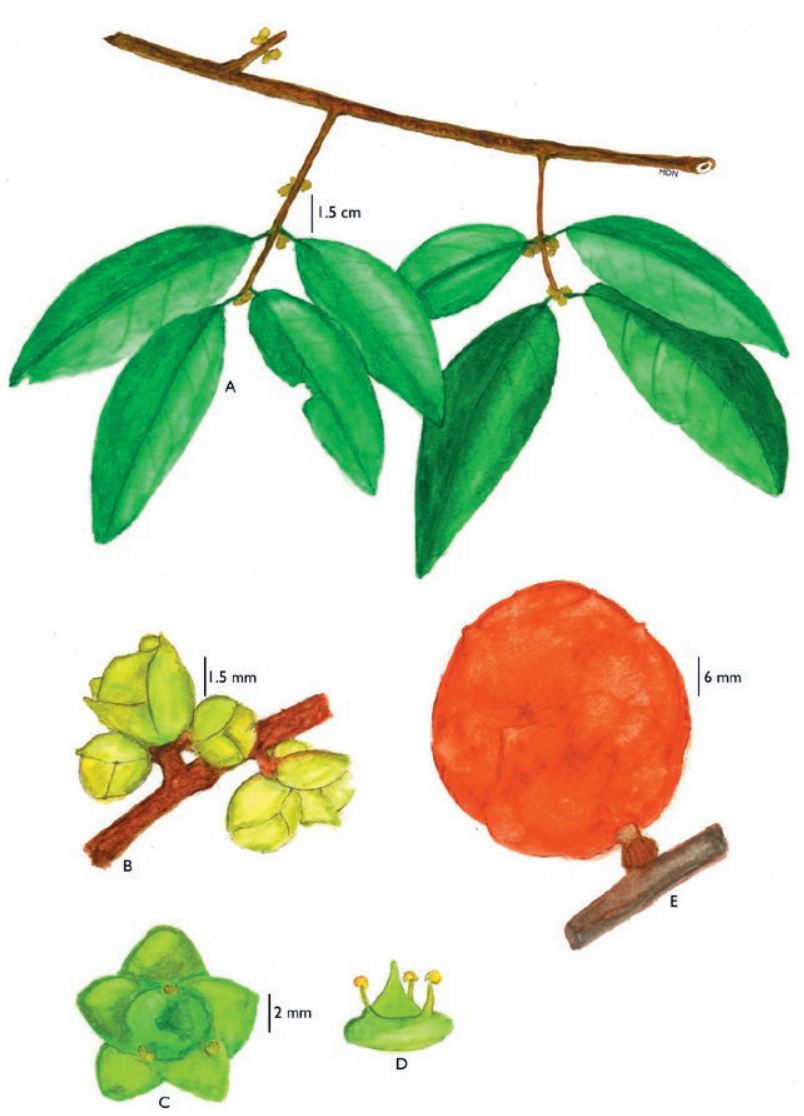

Figure 16. Salacia oblonga (A) flowering twig. (B) Inflorescence. (C) Flower. (D) Disc. (E) Fruit.

inserted in the disk, filaments broad at base, c. $1.5 \mathrm{~mm}$ long, anthers bilobed, pale yellow, ovoid to ellipsoid, thecae confluent longitudinally; ovary embedded in disc, 3-celled, style short, conical. Fruits baccate, globose or sub-globose, $2-5 \mathrm{~cm}$ in diameter, supported on persistent calyx, smooth, rarely uneven or tuberculate, exocarp bright orange-red when ripe, endocarp mucilaginous, 2-8-seeded; seeds 2-8, angular, deltoid, c. 2.7 $\times 1.5 \mathrm{~cm}$, ochreous or brown.

\section{Phenology}

Flowers between January to March, fruits may remain till end of July.

\section{Distribution and habitat}

Peninsular India (Goa, Karnataka, Kerala, Maharashtra, and Tamil Nadu) and Sri Lanka. Semi-evergreen forest, fringing forest, undergrowth forest, along the plateaux borders. The species occurrence in southeast Asia by Kamat et al. (2020) is erroneous. 
Vernacular name

$$
\text { "ponkoranti" (Malayalam). }
$$

\section{IUCN status}

Though the species is fairly widely distributed in peninsular India and Sri Lanka, an IUCN Red list category of Vulnerable (VU) [A2cd] is assigned to it (Ved et al. 2015), mainly due to decline in area, extend and habitat quality caused by over exploitation, agricultural invasion, and road construction. Nevertheless, the species needs to be reassessed based on the precise data on population and threat for the Sri Lankan part of its distribution. The Endangered (EN) category assessed by Kamat et al. (2020) is not supported. This is one of the most exploited species from the wild due to its use as an antidiabetic medicine. As the root are richer in the active compound, salacinol, mature plants are often uprooted from the wild. Salacia oblonga is in need of harvest management to ensure sustainability, as well as a mass cultivation programme as an immediate conservation measure.

\section{Specimens examined}

INDIA. Karnataka, North Kanara, Mundele, May 1853, Ritchie 1653 (K); North Kanara District, Anshi Ghats, 30 Jan. 1886, W. A. Talbot 1378 (K, CAL); ibid, s. d., W. A. Talbot 2931 (BSI); ibid, 22 Dec. 1892 s. coll. 2932 (BSI); ibid, s.dat., W. A. Talbot 2933 (BSI); ibid, 1889, W. A. Talbot 2934 (BSI); Shimoga, Agumbe, Jogi Gundi Falls, 21 Feb. 1963, R. Raghavan 86431 (BSI); Agumbe, 26 Apr. 1997, K. Ravikumar, S. P. Subramani \& P. S. Udayan 11898 (FRLH); North Kanara, s. loc. June 1883, W. A. Talbot 1241 (CAL); North Kanara, Supa, May, T. D. Bell 6043 (CAL); Coorg, Thalacavary, 13 May 2010, P. S. Udayan et al. 6834 (CMPR). Goa, s. l., 29 May 1905, R. K. Bhide s. n. (BSI); Caranzol, 17 Apr. 1966, P. J. Cherian 107165 (BSI); Valpoi, 22 May 1964, K. C. Kanodia 96401 (BSI). Malachi Rai, 22 Mar. 1964, K. C. Kanodia 96401 (CAL); Molem, 19 Apr. 2017, D.C. Jadhav 1465 (NGCPR). Kerala, Kozhikode, Moodadi, 14 Mar. 1996, P. Chiranjit 129 (K); Thiruvananthapuram, on the way to Thekkumalai, 13 Apr. 2009, Devipriya M.S. \& Regy Yohannan $55(\mathrm{MH})$; Thrissur, Vellanipacha, 4 Apr. 2004, P. S. Udayan, 2519 (CMPR); Vellanipacha, 23 Feb. 2007, P. S. Udayan et al. 00104 (CMPR); ibid, 3 Apr. 2004, P. S. Udayan et al. 2489, 2490 (CMPR); ibid, 23 Feb. 2005, P. S. Udayan et al. 3373 (CMPR); ibid, 21 Jan. 2006, P. S. Udayan et al. 3973 (CMPR); ibid, 23 Feb. 2007, P. S. Udayan et al. 4944 (CMPR); Kozhikode, Kakkayam, 26 Mar. 2008 P. S. Udayan 4900 (CMPR); Malappuram, Kot- takkal, Herb Garden, 15 Jan. 2007, P. S. Udayan et al. 5029 (CMPR); ibid, 26 Mar. 2016, D. C. Jadhav 1455 (NGCPR); Kollam, Ventilakara, 7 May 2015, G. Ravikumar 72842 (TBGRI); Thiruvananthapuram, Palode, 28 Dec. 2011, S. M. Shareef 70672 (TBGRI); JNTBGRI Garden, 13 Feb. 2014, S.M. Shareef 79228 (TBGRI); ibid, 16 Feb. 2017, D.C. Jadhav 1462 (NGCPR). Maharashtra, Bombay, s. l., N. A. Dalzell s. n. (K); North Concan, s. loc., Law s. n. (K). Tamil Nadu, Wallich Cat. no. 4227, Herb Wight s.n. (K [K0001038436]; CAL [sheet no. 87592]); Tirunelveli Ghats, Dec. 1871, R. H. Beddome 202 (K); Courtallum, July 1835, R. Wight 464 (K); Nilgiris, Kallar, Oct. 1889, J. S. Gamble $21457(\mathrm{~K})$; Kanyakumari, on the way to Thekkumalai Estate to Courtallum Forest, 20 Feb. 1983, E. Vajravelu, 76587 (MH); ibid, 9 Oct. 2002, C. Murgan, 114184 (MH); Way to Pothigaimalai, 20 Apr. 1992, $R$. Gopalan 99346 (MH); Tirunelveli, on the way to Kongupatty to Puliyara, 10 Oct. 2002, C. Murgan, 114189 (MH); Tirunelveli District, Courtallam, Honey Fall, K. Subramanyam 5606 (MH); Courtallam, s.d., R. H. Beddome 2177 (MH); Tirunelveli, Nellai Kattabomman, 8 Mar. 1994, S. P. Subramani 00320 (FRLH); Tirunelveli, Courtallum, 3 Dec. 2015, M. Alister 88251 (TBGRI). SRI LANKA. Trincomalee, s.d., Herb Wight s.n. (MH [MH1915]); Jaffna, Northern Province, Ampan, 12 Sep. 1974, L. H. Cramer 4336 (MH).

\section{Note}

Udayan et al. (2014) described S. oblonga var. kakkayamana Udayan \& Raghu, but I failed to locate the type of this intraspecific taxon at CAL and MH (Udayan et al. 27802). The specimen collected at the type locality (Udayan et al. 110820: FRLH) has a fruit that matches that of S. oblonga. Hence this variety is here treated as conspecific with $S$. oblonga, which it also resembles in other characters such as a woody scandent habit, subsessile urceolate flowers in axillary branched cymes, elliptic-oblong or oblanceolate leaves, oblong petals, flat disk, globose and bright orange red or yellowish orange fruits.

Londhe (2000) in Flora Maharashtra listed S. oblonga from Sindhudurg District, but during the present study I could not locate the specimens on which this distribution is based. Instead, I saw Wight, Dalzell, Law and Ritchie's collections of S. oblonga from Tamil Nadu (the then Madras Presidency), Karnataka and Maharashtra (the then Bombay Presidency). These were misidentified as S. macrophylla by Ding Hou (1964).

Jadhav 1462 (NGCPR) from the TBGRI campus, Kerala, differs from the rest of the Indian material seen in having noticeably pentamerous and almost sessile flowers. 
10. Salacia wayanadica Sujana, Nagaraju, Ratheesh \& Anil Kumar in Taiwania 60(2): 91. 2015. (Figure 17)

Type: India, Kerala, Wayanad, Periya, Chandanathode, evergreen forests, 853m, 28 Feb. 2011, K. A. Sujana 0401 (holotype CAL! barcode CAL0000025311; isotype MH).

\section{Description}

Straggling shrub or spreading liana up to $2(-5) \mathrm{m}$ high; branches terete, coiled, lenticellate to smooth. Leaves opposite, exstipulate, petiolate; petiole $0.8-1$ $\mathrm{cm}$ long; lamina oblong-elliptic, $8-18 \times 3-6 \mathrm{~cm}$, apex obtuse, base cuneate to rounded, margin entire, coriaceous, glaucous, lateral nerves 8-12 pairs. Inflorescences axillary or ramiflorous, 2-12-flowered, tuberculate fascicle; pedicels $10-12 \mathrm{~mm}$ long, terete, pruinose; bracts inconspicuous, ovate, sepaloid; sepals fused at base, lobes ovate, c. $0.5 \times 1 \mathrm{~mm}$, apex densely bearded, otherwise glabrous; petals c. $2 \times 1.5 \mathrm{~mm}$, oblong, apex acute to hooded, pale green tinged with orange, margin hyaline to yellowish, glabrous; disk annular, pulvinate at base, yellowish orange; stamens 3, incurved (before anthesis); filaments short, anthers bi-lobed, globose, yellow, thecae confluent longitudinally; style raised on the disc, stigma inconspicuous, ovary 3 -celled. Fruits baccate, globose to ovoid, 2-5 $\times 2-35-6$, exocarp orange, thick, tuberculate, endocarp mucilaginous, 3 - or 4-seeded; seeds discoid or ovoid, c. $2 \times 1.5 \mathrm{~cm}$, ochraceous.

\section{Phenology}

Flowers and fruit collected from January to August.

\section{Distribution and habitat}

Endemic to the Western Ghats of Kerala (Figure 19). Climbing over small trees in semi evergreen forest, fringing forest, and along roads.

\section{Specimens examined}

INDIA. Kerala, Wayanad, Chandanthode, 04 Apr. 2018, D. C. Jadhav \& M. D. Nandikar 1483 (NGCPR); ibid, 20 Mar. 1980, V. S. Ramachandran 66831 (MH); ibid, 12 Aug. 1979, V. S. Ramachandran 63921 (MH); ibid, V. S. Ramachandran 63920 (CAL); Wayanad, on the way Periya to Chandanthode, 16 June 1979, V. S. Ramachandran 626321 (CAL); Valady, Periya, Mananthavady, Wayanad, 27 May 1995, Bija \& Joy 10237 (FRLH).

\section{IUCN status}

The species is recorded from only two localities in Wayanad District. Both the localities are adjacent to

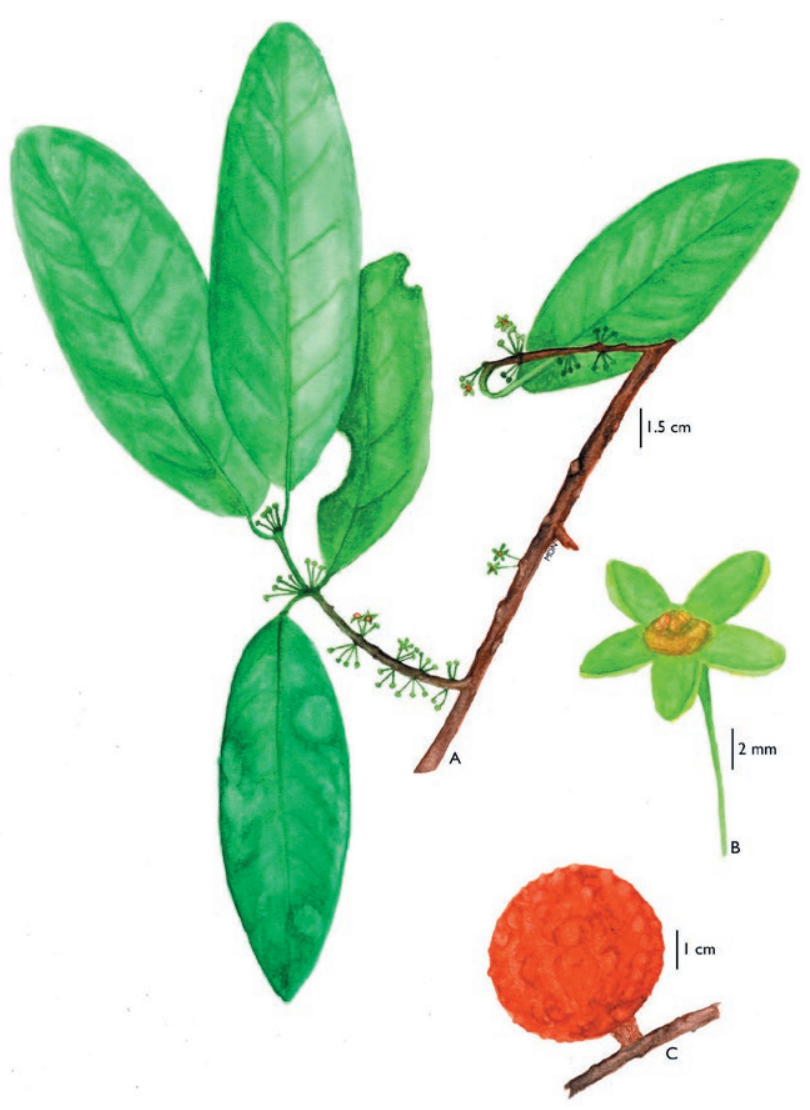

Figure 17. S. wayanadica (A) Flowering twig. (B) Flower. (C) Fruit.

places of human habitation and threatened by habitat degradation. Few plants were found damaged, perhaps due to road clearing and widening, also confirmed by Sujana et al. (2015). The restricted AOO and EOO, continuing decline (observed) of quality of habitat and continuing decline (inferred) in the number of mature individuals suggests an assessment of Critically Endangered (CR) for this species [B1+2ab(iii,v); D].

Note

S. wayanadica shares a similar habit and inflorescence with S. macrosperma, S. gambleana and S. malabarica. However, S. macrosperma differs by having acute to acuminate leaves, laciniate to fimbriate sepals and ovate to broadly elliptic petals. Similarly, S. gambleana differs by having oblanceolate leaves, whereas $S$. malabarica has serrate leaves, much longer pedicels, and glabrous sepals.

\section{EXCLUDED SPECIES}

1. Salacia macrophylla Blume, Bijdr. Fl. Ned. Ind. 5: 221. 1825. 


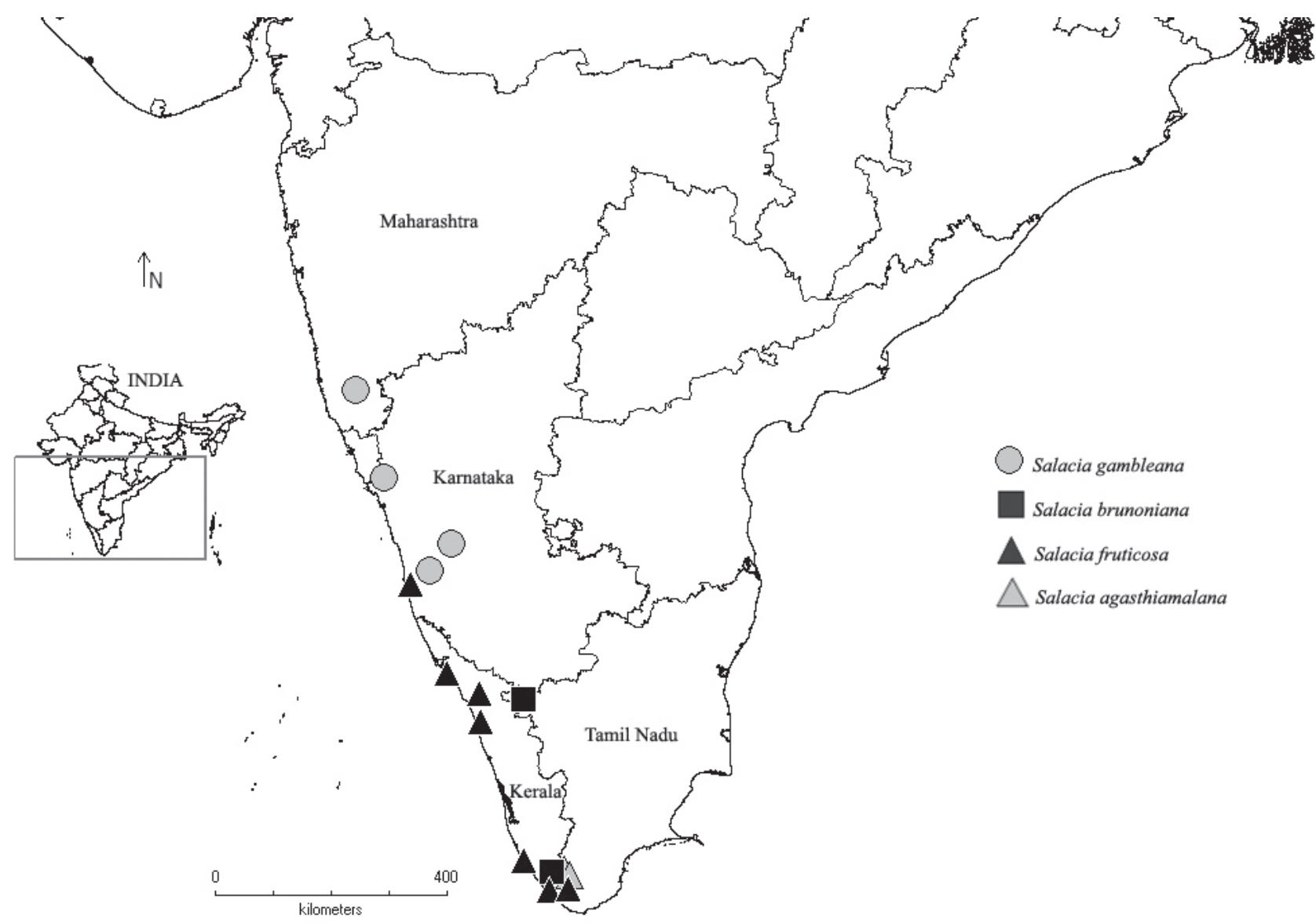

Figure 18. Distribution map of Salacia species endemic to peninsular India.

Type: Indonesia, Java, s.loc. s.d., Herb. Lugd. Bat., Blume 1699 (L! barcode L0015423), lectotype here designated.

(=) Salacia ovalis M.A. Lawson in Fl. Brit. India 1(3): 627. 1875. non Korthals, Flora 31: 579. 1848.

Type: Malaysia, Malacca, s. loc., s. d., A.C. Maingay, 400 (lectotype K! barcode K000669976; isolectoype CAL! barcode CAL0000007497), designated here.

\section{Distribution and habitat}

India (Andaman and Nicobar Islands), Cambodia, Thailand, Hainan. Low land coastline forest.

Note

Blume (1825) described the species from Indonesia. Later, Ding Hou (1964) erroneously reported the occurrence of this species in peninsular India. It seems he misidentified the collections of S. oblonga (from Maharashtra and Karnataka, the then Bombay Presidency) by Dalzell, Law and Ritchie that are available at K. Subsequent authors, Ramamurthy \& Naithani (2000) and Londhe (2000) included the taxon in Maharashtra following Ding Hou (1964). I could not locate any specimen of $S$. macrophylla to support its occurrence in mainland India. The original material collected by Blume and Maingay of S. macrophylla and S. ovalis is housed at $\mathrm{L}, \mathrm{K}$ and CAL. For both the names there is no precise indication of type and hence warrant typification. Comparing the original material with protologue, the best specimen is designated here as lectotype following ICN Art. 9.3 (Turland et al. 2018).

\section{Salacia reticulata Wight, Ill. Ind. Bot. 1: 134. 1838.}

Type: Sri Lanka, s. loc., s. d., Colonel Walker s. n. (K! barcode K000669988), lectotype designated by Wadhwa (1996).

\section{Distribution and habitat}

India (Andaman and Nicobar Islands) and Sri Lanka. Evergreen forest. 


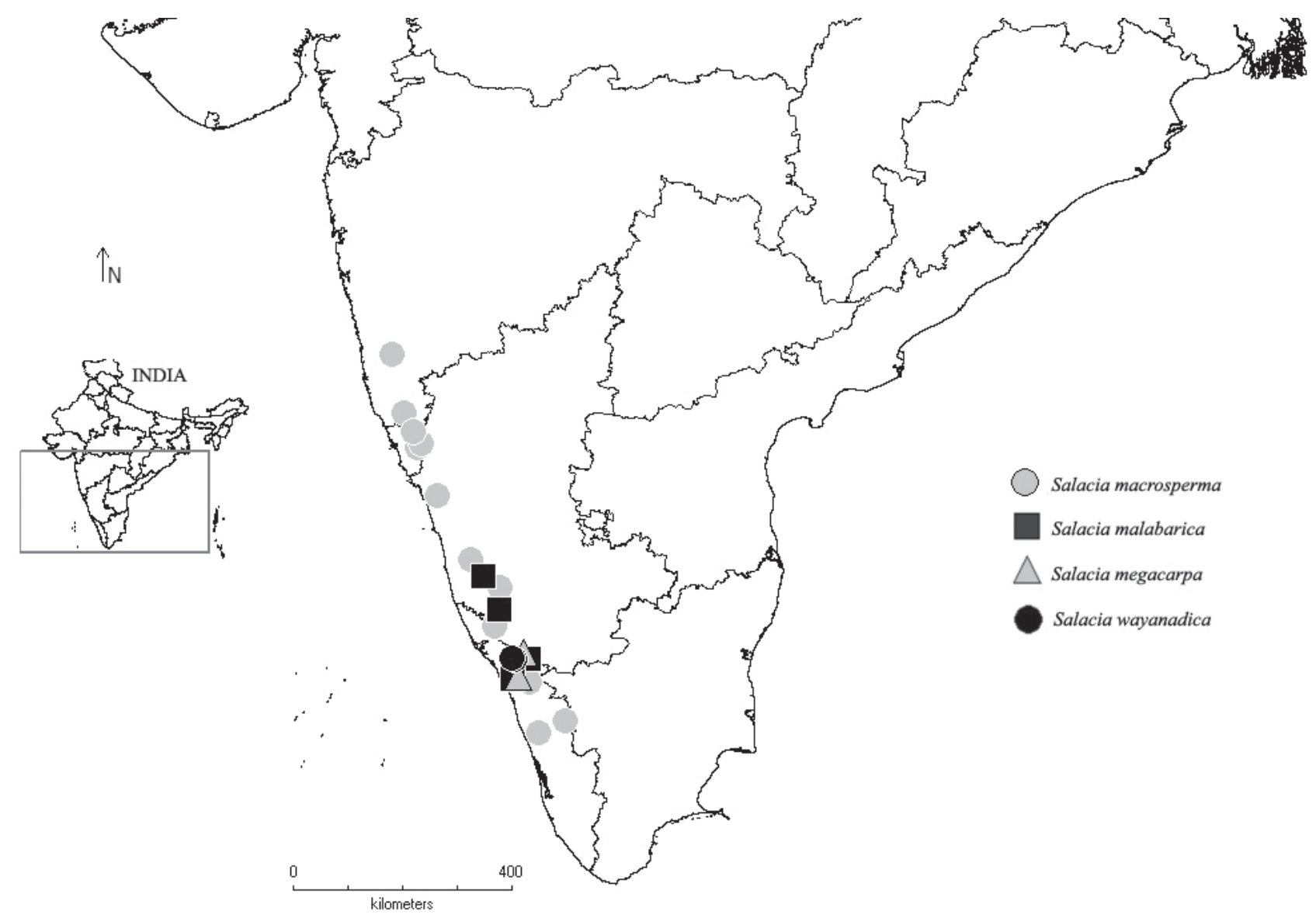

Figure 19. Distribution map of Salacia species endemic to peninsular India.

Note

Wight (1838) described the species based on Walker's collection from Sri Lanka. He was not certain about its occurrence in Malabar of South India. In the protologue, he mentioned that "the identity of the Malabar specimen is doubtful as flowers were not seen, leaves are less distinctly reticulate" and added characters of the fruit is doubtful. Gamble (1918) listed S. reticulata in Madras Presidency based on Rama Rao's collection (CAL 87440). Further details on the doubtful occurrence of $S$. reticulata in India and misapplication of $S$. reticulata to different elements by various authors in India can be found in Udayan and Pradeep (2012). The occurrence recorded in peninsular India by Kamat et al. (2020) is an error.

\section{ACKNOWLEDGMENTS}

I am thankful to Mr. Vijay M. Crishna, Director, Naoroji Godrej Centre for Plant Research (NGCPR), for his encouragement and the Pirojsha Godrej Foun- dation (PGF) for funding the research and Kerala Forest Department for the necessary permissions. I thank Dr. Manoj Lekhak (SUK) and Dr. Ritesh Choudhary (AMHA) for their editing of the manuscript, Mr. Maniruddin Dhabak for his help in the preparation of maps and IUCN Red List assessments, Mr. Arun Prasanth, and Ms. Durga Jadhav for their assistance in writing and field collections, and Dr. Julio Lombardi (UNESP) and anonymous reviewers for their suggestions, improvements to and reviewing of the text. I am also grateful to all the officials of BSI, CAL, CALI, CMPR, FRLH, K, $\mathrm{MH}, \mathrm{RHT}$ and TBGRI for allowing me to consult their Salacia collection.

\section{REFERENCES}

Adanson M. 1763. Familles des Plantes (Adanson). Paris: Vincent; p. 545.

Almeida MR. 1996. Flora of Maharashtra. Vol. 1. Blatter Herbarium, St. Xavier's College, Mumbai: p. 247. 
Almeida SM. 1990. Flora of Savantwadi. Vol. 1. Jodhpur: Scientific Publishers; p.

Bentham G, Hooker JD. 1862. Genera Plantarum. 1(1): 357-360. London.

Blume CL. 1825. Hippocrateaceae: Bijdragen tot de flora van Nederlandsch Indie. 5: 220-221. Batavia: Ter Lands Drukkeji.

Coughenour JM, Simmons MP, Lombardi JA, Cappa JJ. 2010. Phylogeny of Celastraceae subfamily Salacioideae and tribe Lophopetaleae inferred from morphological characters and nuclear and plastid genes. Systematic Botany. 35: 358-366.

Coughenour JM, Simmons MP, Lombardi JA, Yakobson K, Archer RH. 2011. Phylogeny of Celastraceae subfamily Hippocrateoideae inferred from morphological characters and nuclear and plastid genes. Molecular Phylogenetics and Evolution. 59: 320-330.

Dalzell NA, Gibson A. 1861. The Bombay Flora. Bombay: Education Society's Press; 33 p.

Dandy JE. 1969 Nomina Conservanda Proposita: (275) 4662. Salacia Linnaeus. Taxon. 18: 468.

Ding Hou. 1963. Florae Malesianae precursors XXXIV. Notes on some genera of Celastraceae in Malaysia. Blumea. 12: 31-38.

Ding Hou. 1964. Celastraceae. In: Steenis CGGJV, editor Flora Malesiana. 6(3): 389-421. Leiden.

Forman L, Bridson D. 1992. The Herbarium Handbook. Royal Botanic Gardens, Kew; p. 40-63.

Gamble JS. 1916. Decades Kewensis: Plantarum Novarum in Herbario Horth Regii. Bulletin of Miscellaneous Information Kew. 5: 131-136.

Gamble JS. 1918. Flora of the Presidency of Madras. 1(2): 214-215. Adlard and Sons Ltd., London.

Hallé N. 1962. Monographie des hippocrateacae d'Afrigue Occidentale [Monograph of the Hippocrateaceae of West Africa]. Mémoires de l'Institut Français d'Afrique Noire. 64: 151.

Hedin JPT. 1999. Systematic studies of the Neotropical species of Salacia L. (Hippocrateaceae) and its relatives. [Unpublished Ph.D. Thesis], Washington University, Saint Louis.

IUCN Standards and Petitions Committee (2019) Guidelines for Using the IUCN Red List Categories and Criteria. Version 14. Prepared by the Standards and Petitions Committee. Downloadable from http:// www.iucnredlist.org/documents/RedListGuidelines. pdf.

Jadhav DC, Prabhugaonkar, AV, Nandikar MD. 2016. Nomenclature notes on the genus Salacia L. (Celastraceae) in India. Phytotaxa. 265 (2): 121-130.

Kamat SG, Vasudeva R, Patil CG. 2020. Taxonomic identity, occurrence of six species of Salacia and first report on chromosome numbers of the Salacia chinensis L. and Salacia oblonga Wall. ex Wight \& Arn. from Western Ghats of Karnataka (India). Genetic Resources and Crop Evolution. 67: 241-255.

Kurz S. 1872. New Barmese Plants. Journal of the Asiatic Society of Bengal. 41(2): 291-317.

Kurz S. 1875. Descriptions of new Indian Plants. Journal of the Asiatic Society of Bengal. 44 (2): 203.

Lakshminarasimhan P, Srivastava SK. 1993. Salacia korthalsiana Miq. (Celastraceae): an addition to the Indian Flora from Nicobar Islands. Indian Journal of Forestry. 116(1): 77-78.

Lakshminarasimhan P, Ray LN. 1994. Salacia tortuosa Griff. (Celastraceae) - An extended distribution from Andaman Islands, India. Indian Forester. 120: 66-68.

Lawson MA. 1875. Celastraceae. In: Hooker JD, editor The Flora of British India. 1: 625-629. L.Reeve \& Co., London.

Lombardi, JA. 2014. Celastraceae (Hippocrateoideae e Salacioideae). Flora Neotropica Monograph. 114: 1-240.

Londhe AN. 2000. Celastraceae. In: Singh NP, Karthikeyan S, editors Flora of Maharashtra State (Dicotyledones). 1: 527-529. Botanical Survey of India, Kolkata.

Mabberley DJ. 2017. The Plant Book. A portable dictionary of the vascular plants. $4^{\text {th }}$ edition. Cambridge: Cambridge University Press; p. 817.

Majid BN, Sampath KKK, Prakash HS, Geetha N. 2016. Rapid mass propagation of Salacia chinensis L. and Endangered, valuable medicinal plant through direct organogenesis. Indian Journal of Science and Technology, 9(4): 1-8.

Mishra DK, Singh NP. 2001. Endemic and Threatened Flowering Plants of Maharashtra. Dehradun: Botanical Survey of India; p. 76.

Moat J. 2007. Conservation assessment tools extension for Arc View 3.x, version 1.2. GIS Unit, Royal Botanic Gardens, Kew. http://www.rbgkew.org.uk/gis/cats

Nicolson DH, Suresh CR. 1986. (813) Proposal to Conserve Salacia against Courondi (Hippocrateaceae). Taxon. 35(1): 181-182.

Patwardhan A, Pimputkar M, Joshi R. 2014. Evaluation of anti-diabetic property of extracts of different plant parts of Salacia chinensis Linn. Journal of Biodiversity, Bioprospecting and Development. 1: 107. DOI: 10.4172/ijbbd.1000107

Pelser PB, Barcelona, JF, Nickrent DL, editors. 2016 onwards. Celastraceae. Co's Digital Flora of the Philippines. Available from https://www.philippineplants. org/Families/Celastraceae.html

Purkayastha CS. 1938. Four New Species from Assam. Indian Forester. 64 (5): 277-278. 
Rajasekar C, Silambarasan R, Kottaimuthu R. 2018. Salacia agasthiamalana Udayan, Yohannan \& Pradeep (Celastraceae: Salacioideae): A new record for Tamil Nadu, India. NeBio. 9(3): 245-248.

Ramamurthy K, Naithani BC. 2000. Salacia. In: Singh NP, Vohra JN, Hajara PK, Singh DK., editors, Flora of India. 5: 150-162, Botanical Survey of India, Calcutta.

Rheede HA van. 1683. Hortus Indicus Malabaricus. 4: 103-104, t. 50. Amsterdam.

Robson NKB. 1965. New and little-known species from the Flora Zambesiaca area XVI. Taxonomic and nomenclatural notes on Celastraceae. Boletim da Sociedade Broteriana. 39 (2.ser.): 5-55.

Robson NKB, Hallé N, Mathew B, Blakelock R. 1994. Celastraceae. In: Polhill RM (ed.) Flora of Tropical East Africa. Rotterdam: AA Balkema; Pp. 1-43.

Roxburgh W. 1820 Flora Indica or Description of Indian Plants. 1: 173, Mission Press, Serampore.

Sasidharan N, Anaz KM. 2015. Note on the identity of Salacia vellaniana Udayan, Yohannan \& Pradeep (Celastraceae). International Journal of Advanced Research. 3(4): 386-389.

Shareef SM, Kumar ESS. 2013. Taxonomic note on Salacia brunoniana Wight \& Arn. (Celastraceae): An endangered species of the peninsular India, Indian Journal of Forestry. 36(2): 273-276.

Simmons MP. 2004. Celastraceae. In: Kubitzki K. editor, The families \& genera of vascular plants. 6: 29-64, Berlin Heidelberg: Springer-Verlag.

Simmons MP, Savolainen V, Clevinger CC, Archer RH, Davis JI. 2001. Phylogeny of the Celastraceae inferred from 26S Nuclear Ribosomal DNA, Phytochrome B, rbcL, atpB, and morphology. Molecular Phylogenetics and Evolution. 19(3): 353-366.

Smith AC. 1940. The American species of Hippocrateaceae. Brittonia. 3: 341-555.

Sujana KA, Nagaraju S, Narayana, MKR, Kumar NA. 2015. A new species of Salacia (Celastraceae) from India. Taiwania. 60 (2): 91-94.

Turland NJ, Wiersema JH, Barrie FR, Greuter W, Hawksworth DL, Herendeen PS, Knapp S, Kusber WH, Li DZ, Marhold K, May TW, McNeill J, Monro AM, Prado J, Price MJ, Smith GF (editors). 2018. International Code of Nomenclature for algae, fungi, and plants (Shenzhen Code) adopted by the Nineteenth International Botanical Congress Shenzhen, China, July 2017. Regnum Vegetabile 159. Glashütten: Koeltz Botanical Books.

Udayan PS, Pradeep AK. 2012. Notes on the occurrence of Salacia reticulata (Hippocrateceae) in India. Acta Botanica Hungarica. 54(3-4): 433-441.
Udayan PS, Raghu AV, Sreekumar VB, Muraleedaran EM. 2014. A new variety of Salacia (Celastraceae) from the Western Ghats of Kerala, South India. International Journal of Plant, Animal and Environmental Sciences. 4(3): 100-102.

Udayan PS, Yohannan R, Devipriya MS, Devipriya V, Pradeep AK. 2012. A new species of Salacia (Hippocrateaceae) from South India. Edinburgh Journal of Botany 69 (2): 1-4.

Udayan PS, Yohannan R, Devipriya MS, Devipriya V, Pradeep AK. 2013. Salacia vellaniana Udayan, Yohannan, Pradeep (Celastraceae), a new species from India. Candollea. 68(1): 147-149.

Ved D, Saha D, Ravikumar K, Haridasan K. 2015. Salacia oblonga. The IUCN Red List of Threatened Species: e.T50126639A50131425. http://dx.doi.org/10.2305/ IUCN.UK.2015-2.RLTS.T50126639A50131425.en. Downloaded on 13 November 2019.

Wadhwa BM. 1996. Hippocrataceae. In: Dassanayake MD, Clayton WD (eds.), A Revised Handbook to the Flora of Ceylon. Amerind Publishing, New Delhi 10.

Wight R, Arnott GAW. 1834. Hippocrateaceae. Prodromus Florae Peninsulae Indiae Orientalis. 1: 103-106, Parbury, Allen \& Co., London.

Wight R. 1838. Illustrations of Indian Botany. 1(7): 134, Pharoah JB, Madras.

Wight R. 1840. Hippocrateaceae. Illustration of Indian Botany. 1: 132-134, Pharoah JB, Madras.

Wight R. 1845. Icones Platarum Indiae Orientalis. 3: 3, t. 962, Frank \& Co. \& Ostell, Lepage \& Co., Madras.

Yoshikawa M, Toshiyuki M. Hiromi S. Hisashi M. Johji Y. Genzou T. Osamu M. 1997. Salacinol, potent antidiabetic principle with unique thiosugar sulfonium sulfate structure from the Ayurvedic traditional medicine Salacia reticulata in Sri Lanka and India. Tetrahedron Letters. 38(48): 8367-8370. 